NISTIR 7966

\title{
Security of Interactive and Automated Access Management Using Secure Shell (SSH)
}

Tatu Ylonen

Paul Turner

Karen Scarfone

Murugiah Souppaya

This publication is available free of charge from: http://dx.doi.org/10.6028/NIST.IR.7966

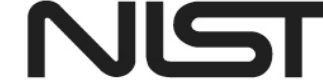

National Institute of Standards and Technology

U.S. Department of Commerce 
NISTIR 7966

\section{Security of Interactive and Automated Access Management Using Secure Shell (SSH)}

Tatu Ylonen SSH Communications Security Helsinki, Finland

Paul Turner Venafi Salt Lake City, UT

Karen Scarfone Scarfone Cybersecurity Clifton, VA

Murugiah Souppaya

Computer Security Division Information Technology Laboratory

This publication is available free of charge from:

http://dx.doi.org/10.6028/NIST.IR.7966

October 2015

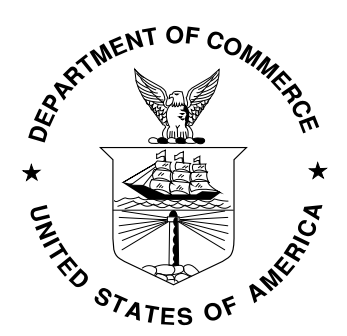

U.S. Department of Commerce

Penny Pritzker, Secretary

National Institute of Standards and Technology Willie May, Under Secretary of Commerce for Standards and Technology and Director 


\title{
National Institute of Standards and Technology Internal Report 7966 50 pages (October 2015)
}

This publication is available free of charge from: http://dx.doi.org/10.6028/NIST.IR.7966

Certain commercial entities, equipment, or materials may be identified in this document in order to describe an experimental procedure or concept adequately. Such identification is not intended to imply recommendation or endorsement by NIST, nor is it intended to imply that the entities, materials, or equipment are necessarily the best available for the purpose.

There may be references in this publication to other publications currently under development by NIST in accordance with its assigned statutory responsibilities. The information in this publication, including concepts and methodologies, may be used by Federal agencies even before the completion of such companion publications. Thus, until each publication is completed, current requirements, guidelines, and procedures, where they exist, remain operative. For planning and transition purposes, Federal agencies may wish to closely follow the development of these new publications by NIST.

Organizations are encouraged to review all draft publications during public comment periods and provide feedback to NIST. All NIST Computer Security Division publications, other than the ones noted above, are available at http://csrc.nist.gov/publications.

\section{Comments on this publication may be submitted to:}

\author{
National Institute of Standards and Technology \\ Attn: Computer Security Division, Information Technology Laboratory \\ 100 Bureau Drive (Mail Stop 8930) Gaithersburg, MD 20899-8930
}




\title{
Reports on Computer Systems Technology
}

The Information Technology Laboratory (ITL) at the National Institute of Standards and Technology (NIST) promotes the U.S. economy and public welfare by providing technical leadership for the Nation's measurement and standards infrastructure. ITL develops tests, test methods, reference data, proof of concept implementations, and technical analyses to advance the development and productive use of information technology. ITL's responsibilities include the development of management, administrative, technical, and physical standards and guidelines for the cost-effective security and privacy of other than national security-related information in Federal information systems.

\begin{abstract}
Users and hosts must be able to access other hosts in an interactive or automated fashion, often with very high privileges. This is necessary for a variety of reasons, including file transfers, disaster recovery, privileged access management, software and patch management, and dynamic cloud provisioning. Accessing other hosts is often accomplished using the Secure Shell (SSH) protocol. The SSH protocol supports several mechanisms for interactive and automated authentication. Management of this access requires proper provisioning, termination, and monitoring processes. However, the security of SSH keybased access has been largely ignored to date. This publication assists organizations in understanding the basics of SSH interactive and automated access management in an enterprise, focusing on the management of SSH user keys.
\end{abstract}

\section{Keywords}

access control; authentication; automated access management; device authentication; interactive access management; Secure Shell (SSH); user authentication

\section{Acknowledgments}

The authors wish to thank their colleagues who reviewed drafts of this document and contributed to its technical content.

\section{Trademark Information}

All registered trademarks or trademarks belong to their respective organizations. 


\section{Table of Contents}

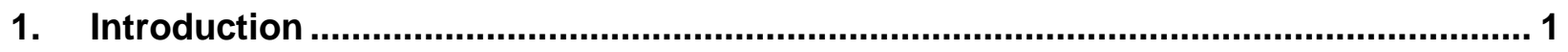

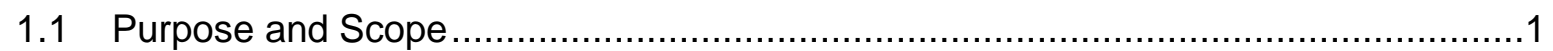

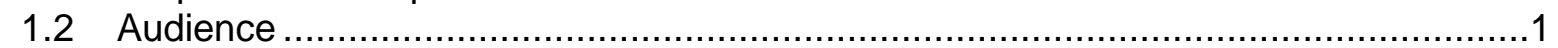

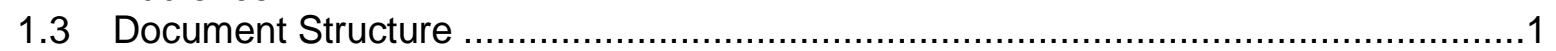

2. The Basics of Access Management and Automated Access Management ................. 2

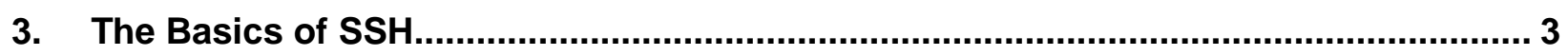

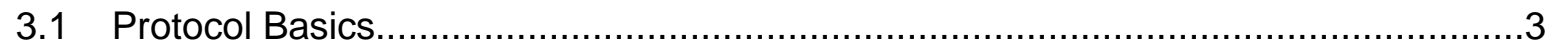

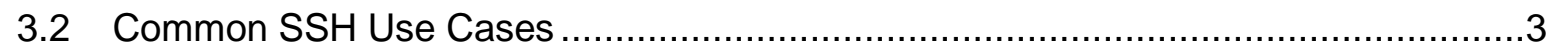

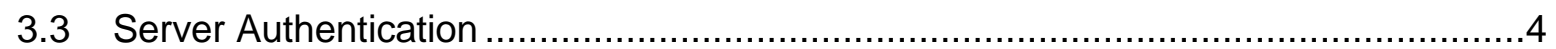

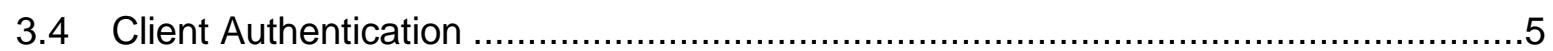

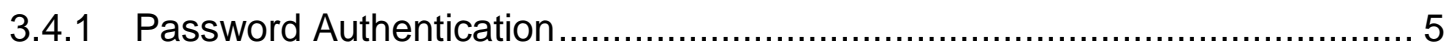

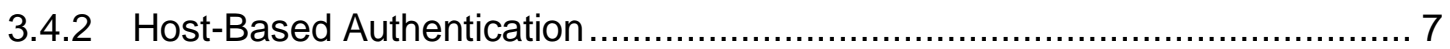

3.4.3 Kerberos Authentication ............................................................... 7

3.4.4 Public Key Authentication................................................................. 9

3.4.5 User Authentication Summary ....................................................... 11

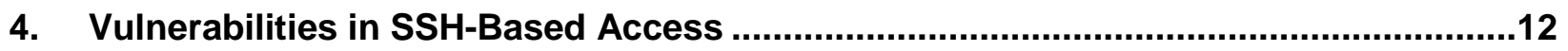

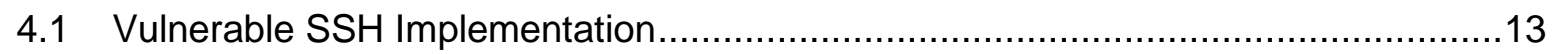

4.2 Improperly Configured Access Controls .................................................13

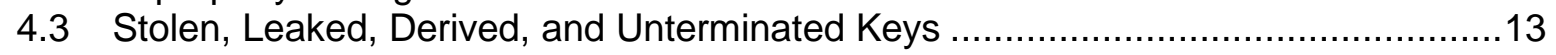

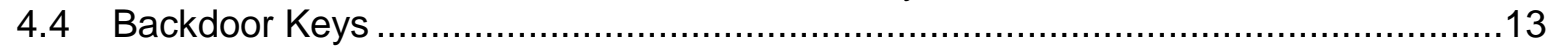

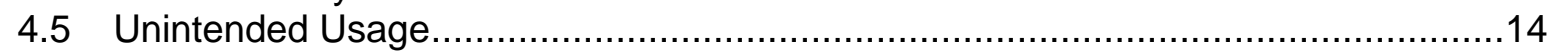

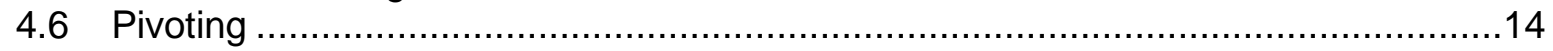

4.7 Lack of Knowledge and Human Errors ........................................................... 14

5. Recommended Practices for Management.........................................................15

5.1 SSH Security Policies and Procedures ...................................................... 15

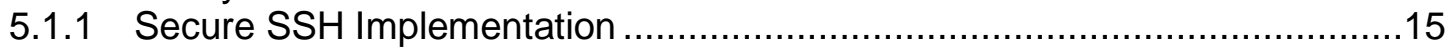

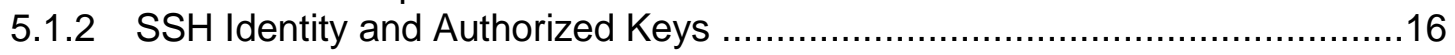

5.2 SSH Key-Based Access Provisioning, Life Cycle, and Termination Processes .......17

5.3 Establish Continuous Monitoring and Audit Processes ......................................19

5.4 Inventory and Remediate Existing SSH Servers, Keys, and Trust Relationships .....20

5.5 Automate Processes ........................................................................... 21

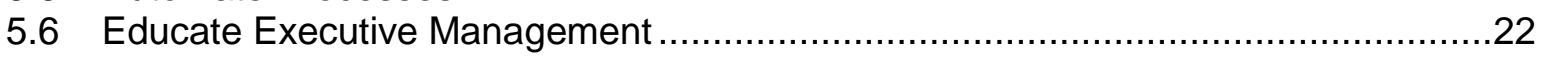

6. SSH-Based Access Management Planning and Implementation................................23

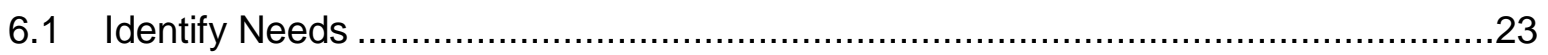

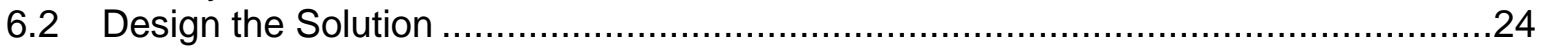

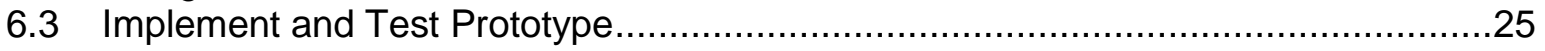

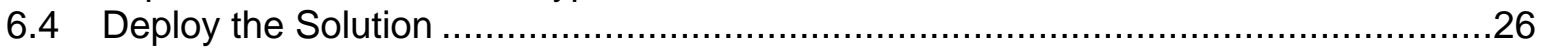

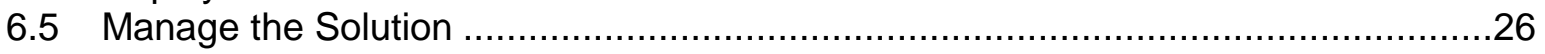

7. Solution Planning and Deployment .....................................................................28

Appendix A- NIST SP 800-53 Controls Mapping ..........................................................29

Appendix B-Cybersecurity Framework Subcategory Mapping ….................................34

Appendix C - SSH Key Management Tool Selection .......................................................36 
Appendix D- Acronyms and Abbreviations ..........................................................40

Appendix E- Glossary ..................................................................................................41

Appendix F- References...................................................................................4 


\section{Introduction}

\subsection{Purpose and Scope}

The purpose of this document is to assist organizations in understanding the basics of Secure Shell (SSH) and SSH access management in an enterprise, focusing on the management of SSH user keys.

\subsection{Audience}

This document is for security managers, engineers, administrators, and others who are responsible for planning, acquiring, testing, implementing, and maintaining SSH solutions. Portions of the document may be of interest to executives and SSH users.

\subsection{Document Structure}

The remainder of this document is organized into the following sections and appendices:

- Section 2 discusses the basics of access management and automated access management.

- Section 3 examines the basics of SSH.

- Section 4 describes the primary categories of vulnerabilities in SSH user key management.

- Section 5 lists recommended practices for SSH-based access management.

- Section 6 explains planning and implementation processes for SSH-based access management.

- Section 7 discusses solution planning and deployment.

- Appendix A provides a mapping to NIST Special Publication (SP) 800-53 Revision 4 security controls. ${ }^{1}$

- Appendix B provides a mapping to Cybersecurity Framework Version 1.0 subcategories.

- Appendix C lists criteria for selecting and procuring tools for managing SSH keys.

- Appendix D defines selected acronyms and abbreviations used in the document.

- Appendix E defines selected terms used in the document.

- Appendix F lists references.

1 NIST SP 800-53 Revision 4, Security and Privacy Controls for Federal Information Systems and Organizations, April 2013 (updated 1-22-2015), http://dx.doi.org/10.6028/NIST.SP.800-53r4 


\section{The Basics of Access Management and Automated Access Management}

Controlling access to information systems is critical for information security. Access controls exist on many levels and use many technologies. The levels include physical restrictions on access to hardware; logical controls for accessing network interfaces, hardware management ports on servers, virtualization hypervisors, operating system (OS) user accounts, and information through database systems; and logical controls implemented by applications.

Information security (confidentiality, integrity, and availability) is compromised if controls at any of these levels fail. Breaches at different levels have different implications. Generally, a breach at the hardware, hypervisor, or OS level is more serious than a breach at the application level. For example, breaking into a database account on a server may permit reading, modifying, and destroying any data in a database, bypassing normal database-level controls. Breaking into a "root" (administrator) account generally permits doing this to all data on all accounts on the system, plus installing deeply hidden backdoors, modifying the operating system, corrupting data, or rendering the system unbootable.

Most operating systems use user accounts as the primary unit of access control. In this document, a user account means an OS level user account unless otherwise specified. User accounts correspond to people (including system administrators) and service accounts are used for running application software or are used internally by the OS. It is worth noting that many applications implement their own user accounts that do not correspond to OS level user accounts (they essentially share the service account of the application); however, such application-level accounts are generally beyond the scope of this document.

User accounts may be stored in a centralized repository (e.g., Active Directory [AD] or Lightweight Directory Access Protocol [LDAP]) or may be configured locally on a system. A user account defined locally is generally distinct on each system (separate password, separate home directory, etc.), even if the same account name is used on multiple computers, while user accounts defined in a centralized repository are often available on more than one computer and share the same home directory (on a networked file system). Service accounts are very commonly local accounts, and accounts for people are often stored in a directory.

In a very real sense, access control is the essence of information security. Other security technologies primarily exist to implement and enforce access controls, make it harder to analyze and attack access control systems, limit the impact of actual breaches, evaluate the current state of protections, detect suspicious activity, counteract undesired activity, or help analyze what happened after the fact. The critical balance in information security is between the need to grant access and the need to limit access. Consequently, access must be provisioned (based on proper justification for that level of access) and it must be eventually terminated (e.g., when an employee leaves the role that justified the access, when a client system or application requiring automated access is decommissioned).

Access to hosts has become increasingly automated. Examples of this automation include file transfers, disaster recovery, privileged access management, software and patch management, and dynamic cloud provisioning. This automation involves transferring data and executing commands, such as having hosts reconfigure other hosts. Thus, hosts must access other hosts, often with very high privileges.

Unfortunately, there has been little planning and oversight of automated and interactive machine-tomachine access control. Instead, such access has been added and configured on an ad hoc basis by system administrators, vendors, and integrators as part of other projects, without formal access control lifecycle management (e.g., standardized provisioning and termination processes, access token management (e.g., periodic password changes)). This publication explores the field of SSH-based access management, with a strong focus on security issues and how to best address them. 


\section{The Basics of SSH}

Secure Shell (SSH) is a protocol for securely logging into a remote host and executing commands on that host (e.g., administrative commands). What distinguishes the SSH protocol from earlier remote administration protocols, such as telnet, remote shell (rsh), remote login (rlogin), and remote copy (rcp), is its built-in support for robust security features such as secure user and device authentication and transmission encryption. SSH has almost completely taken the place of these insecure remote administration protocols.

Today, SSH software is natively included and used as the primary remote administration mechanism for many operating systems and devices, including Linux, Unix, routers, firewalls, network appliances, security appliances, and other systems. It is also embedded behind the scenes into a wide variety of Information Technology (IT), networking, and security technologies, including file transfer, systems management, identity management, and privileged access management. In addition to manually remotely connecting to and managing hosts and devices, SSH is used for integrating hosts and automating their operation.

This section examines the basics of Secure Shell (SSH) version 2.0. First, Section 3.1 explores SSH protocol basics. Next, Section 3.2 discusses the most common use cases for SSH, including the use case scenario of interest in this publication: user and automated access. Section 3.3 examines SSH server authentication, while Section 3.4 details SSH client authentication.

\subsection{Protocol Basics}

The SSH protocol has a typical client/server architecture. An SSH client application on host A initiates a connection to an SSH server application on host B. These two hosts negotiate encryption algorithms for their transmissions, then establish a session key and perform device authentication for the server host (host $\mathrm{B})^{2}$, and finally send client (or user) authentication credentials (e.g., username and password) to the server. Assuming that this authentication succeeds, an SSH connection is said to be established between the hosts, ready for use.

The current version of the SSH protocol is 2.0. Earlier versions of the protocol have serious known vulnerabilities that preclude their use. For more information on the formal definition of the SSH protocol version 2.0, see [RFC4251], [RFC4252], [RFC4253], and [RFC4254]. All references to the SSH protocol in this publication are to version 2.0 unless explicitly stated otherwise.

\subsection{Common SSH Use Cases}

There are three common use cases for SSH:

- Interactive use. SSH is used for managing and configuring Unix and Linux computers, networking equipment, and various other types of hosts remotely. SSH is also used for running applications remotely (particularly text-based legacy applications).

- File transfers. SSH is used as the foundation of the Secure Copy (scp) and Secure File Transfer Protocol (SFTP) protocols. These protocols are used to transfer files between hosts while leveraging the security capabilities built into SSH.

2 The primary purpose of authenticating the server is to prevent man-in-the-middle attacks. 
- Point-to-point tunneling. SSH can be used to implement a virtual private network (VPN) tunnel to protect data transmitted between two hosts. One or both of these hosts may be acting as a gateway for other hosts behind it.

This publication covers all of these use cases in the context of user and automated access. Automated access refers to accessing a host from another host in an automated fashion (without human intervention). $\mathrm{SSH}$ is frequently used for automated access for a variety of purposes, including managing large IT environments, integrating applications, and provisioning virtual machines in cloud services.

Automated access is commonly used with functional accounts, system accounts, service accounts, and other non-interactive user accounts (sometimes also called non-user accounts). Such accounts are used by operating systems, server applications (e.g., databases), and other applications for running processes. Automated access is also frequently used for file transfer functions.

Automated access may be unrestricted, allowing any commands to be executed, or may be limited to specific commands or operations, such as file transfers (perhaps limited to a specific directory). Organizations should limit automated access so that only the necessary commands can be executed and only the necessary resources can be engaged.

\subsection{Server Authentication}

The confidentiality and integrity of SSH rely on strong authentication of both the SSH server and client. Authentication of the SSH server by the client is performed with public key cryptography via public keys explicitly trusted by the client or certificates issued by a certification authority — public keys are more commonly used than certificates. ${ }^{3}$

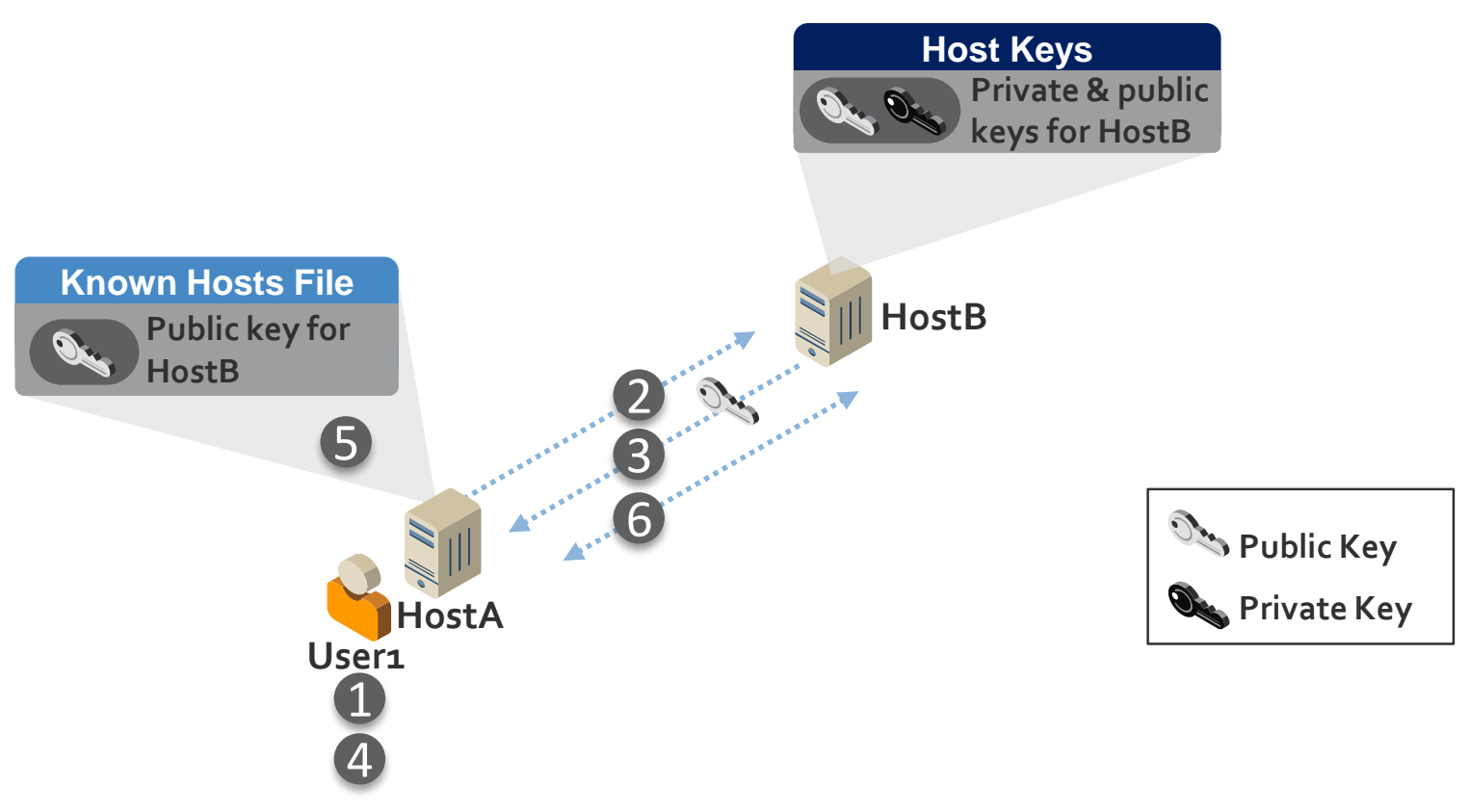

Figure 3-1: Process for Initially Trusting an SSH Server's Public Key

$3 \quad$ X.509v3 compliant host certificates are a useful alternative for host keys in large environments and make host key rotation much easier. 
Figure 3-1 illustrates the process for initially trusting an SSH server's public key:

1. User1 initiates its first login to HostB.

2. HostA connects to HostB.

3. HostB sends its public key to HostA. ${ }^{4}$

4. The public key sent by HostB is displayed for User1. User1 verifies that it is the correct public key for HostB by comparing it against the value received via a different communication channel.

5. HostA stores HostB's public key in User1's known hosts file along with the name of HostB in order to authenticate HostB during future connections without prompting the user to verify that the key is the correct public key for HostB.

6. HostA authenticates HostB using HostB's public key and establishes an encrypted connection with HostB.

Note: If the User1 account is being used by an automated process, the user who reviews the public key on first connection is typically the administrator for the automated process. To avoid this process, the administrator can prepopulate the known hosts file with HostB’s public key.

Care must be taken by users and administrators in ensuring that the correct public keys for each server are trusted as known host keys to prevent man-in-the-middle attacks. In addition, a man-in-the-middle attack can also be launched if the attacker is able to get a copy of the server's private key. Wherever possible, users should have limited ability to change known host key configurations. In addition, known hosts files should be hashed to minimize information available to potential attackers.

\subsection{Client Authentication}

Client authentication refers to the authentication of interactive users (administrators and other users) or automated processes operating on SSH clients. Authentication occurs for a particular account on the server.

The SSH protocol supports several mechanisms for authenticating users, including passwords, host-based authentication, Kerberos, and public key authentication. All these authentication methods fundamentally rely on some secret information, and when used for automated access, this secret information must be stored locally or be otherwise accessible. One or more client authentication methods can be enabled on each SSH server. Each authentication method features pros and cons for security and flexibility-these pros/cons are often different for interactive users and automated process. Organizations must carefully evaluate and select the client authentication method(s) that are approved for use in their environment, disabling others. This section briefly discusses these forms of client authentication, focusing on their relevancy and appropriateness for interactive users and automated processes.

\subsubsection{Password Authentication}

There are two kinds of password authentication mechanisms in SSH: basic password authentication and keyboard-interactive authentication. Basic password authentication is a legacy method defined by the SSH protocol standards. Keyboard-interactive authentication is used in most modern environments, and can support challenge-response authentication and one-time passwords in addition to traditional password authentication. LDAP may be used in lieu of local credential databases (e.g., password files) to reduce the number of passwords users must remember and manage. With both password and keyboard-interactive

$4 \quad$ An SSH server may have more than one public/private key pair if multiple algorithms (e.g., RSA, DSA, ECDSA) are supported on the server. 
authentication, usernames, passwords, and challenge responses are sent from the client (HostA) to the server (HostB) across the encrypted SSH connection. This protects the credentials in transit across the network but they are still subject to compromise in a man-in-the-middle attack. Password authentication is more commonly used for interactive users, but less commonly for automated access, although it is sometimes seen with hard-coded passwords in scripts and management systems.

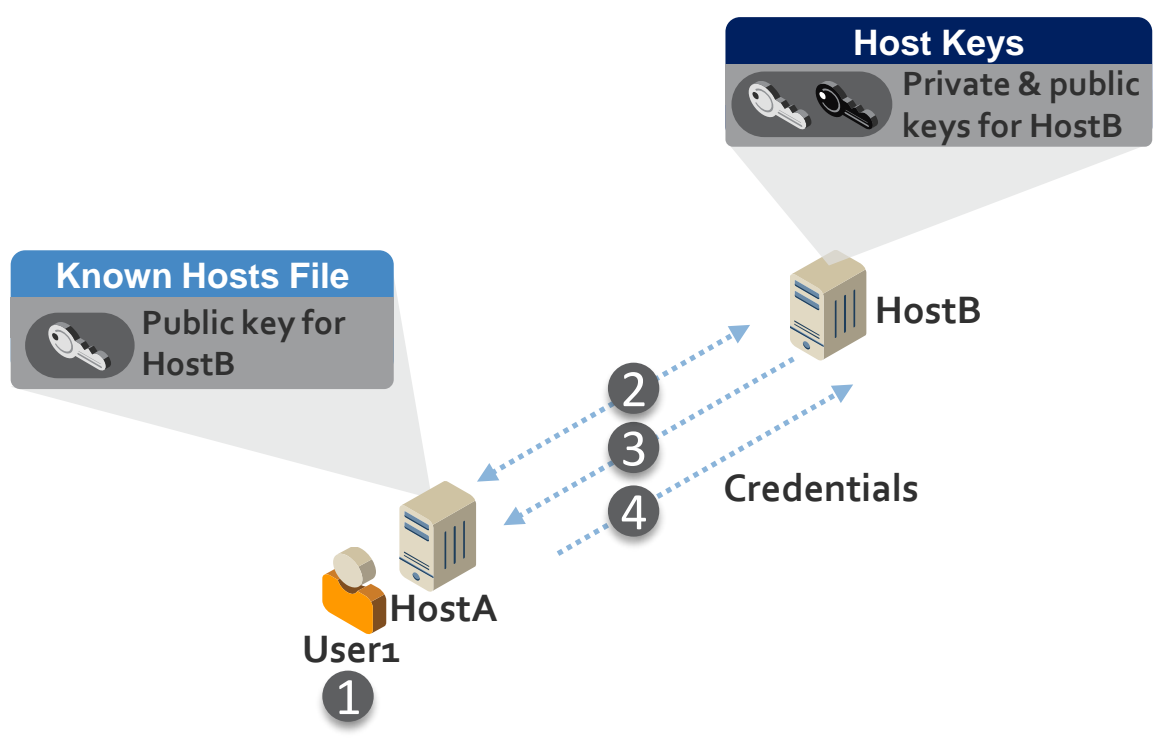

Figure 3-2: Password Authentication Process

Figure 3-2 illustrates the password authentication process:

1. User1 initiates a login to HostB.

2. HostA authenticates HostB using the HostB public key stored in User1's known hosts file and establishes an encrypted connection with HostB. ${ }^{5}$

3. User1 is prompted for a username and password and potentially other credentials (for keyboardinteractive authentication) required by HostB.

4. The credentials are sent to HostB over the encrypted connection. HostB authenticates User1 by verifying the credentials provided by User1.

For interactive users, password authentication provides users mobility, as they can enter their password anywhere from which they have SSH access. However, if interactive users are required to remember and manage different passwords for multiple systems, it can create administrative and security challenges.

Password authentication is generally not recommended for automated processes because it doesn't provide the level of access control available with other authentication methods, especially public key authentication.

If password authentication is used for interactive users or automated access, the passwords should be rotated frequently in accordance with the server organization's password policy (which should also contain requirements such as minimum password length, minimum password complexity, etc.)

5 This step is described above in Server Authentication. It is included here to promote understanding of when server authentication occurs during the password authentication process. 


\subsubsection{Host-Based Authentication}

Host-based authentication uses a server host's (HostA) host key - the key typically used by clients to verify the server's identity - to authenticate that server (HostA) to another server (HostB) and to vouch for the identity of the user (User1) on the client side server (HostA). A configuration file (.shosts) can be used with any user account on the destination server (HostB) to specify which users on which hosts can log into that account without further authentication.

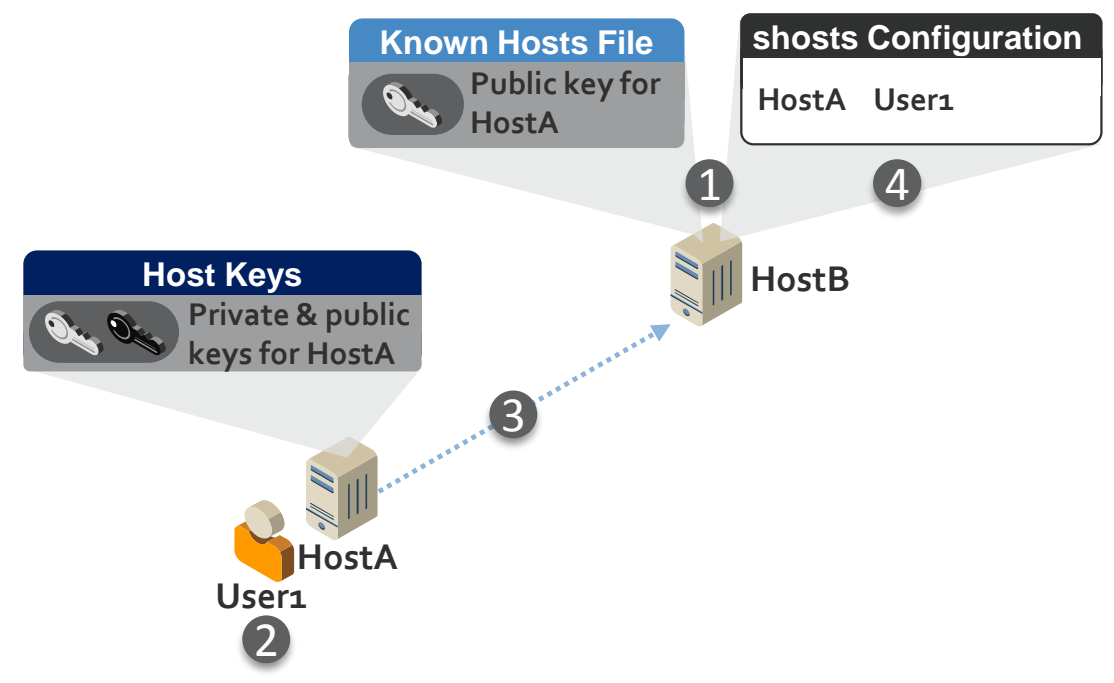

Figure 3-3: Host-Based Authentication

Figure 3-3 illustrates host-based authentication:

1. The administrator for HostB places the public key for HostA in the known hosts file on HostB and configures the shosts (e.g. /.shosts or shosts.equiv) to allow User1 to authenticate from HostA.

2. User1 starts to login to HostB.

3. HostA authenticates to HostB using the host key for HostA.

4. HostB confirms in the shosts configuration that User1 is authorized to access the target account on HostB from HostA.

Host-based authentication does not permit configuring command restrictions-limits on what can be done on the destination server when accessed. Because of this, it is not recommended for automated access. Host-based authentication is not recommended for interactive users because it does not present an interactive login, which is not generally considered a good practice, especially for accounts with elevated privileges.

\subsubsection{Kerberos Authentication}

Kerberos (usually together with an LDAP-based directory, such as Active Directory) implements single sign-on within a Windows domain or Kerberos realm, and allows user accounts and credentials to be stored in a centralized directory. A user authenticates to the directory and then is able to access any account with the same name on SSH-based machines that are joined to the same domain or realm. On one hand, this can be beneficial for single sign-on and a single point for managing user accounts, including 
the ability to disable the account centrally and remove all access. On the other hand, such single sign-on implies that once a user has authenticated to an account using Kerberos, it is possible to log in to any other server that has the same account name and is in the same domain (with Kerberos authentication enabled) without further authentication. This can easily create lots of unwanted implicit trust relationships. Another concern is that currently widely used SSH implementations do not support command restrictions for Kerberos.

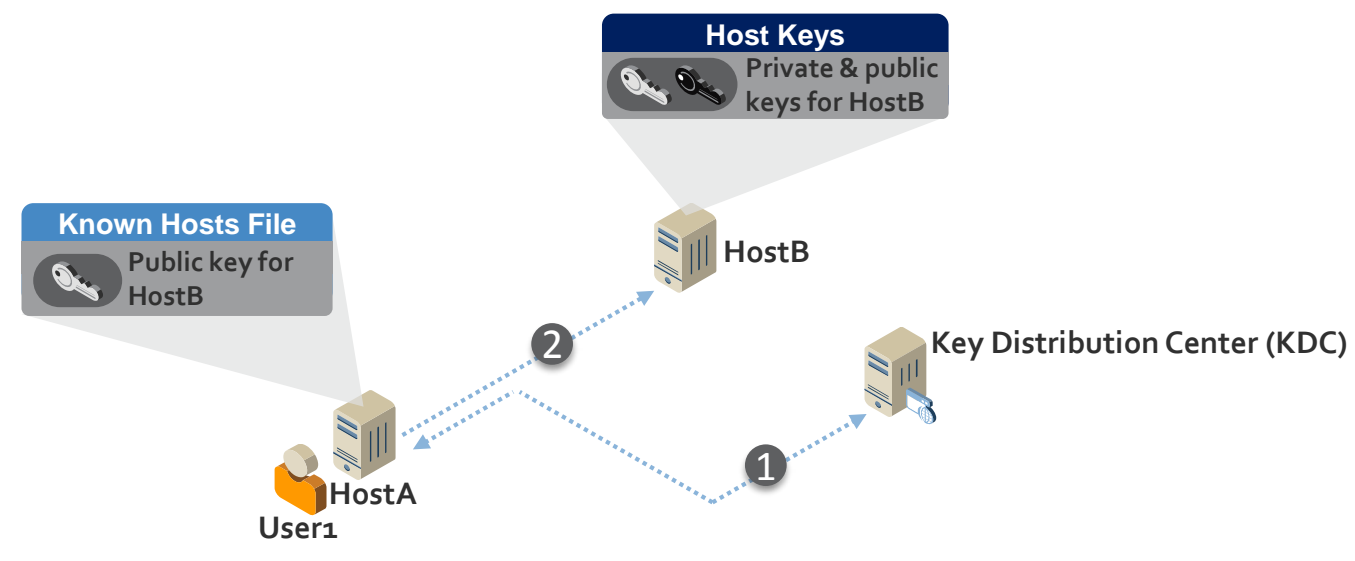

Figure 3-4: Kerberos Authentication

Figure 3-4 illustrates Kerberos authentication with SSH:

1. User1 authenticates to the Kerberos Key Distribution Center (KDC) and receives a ticket, which HostA stores for future authentications.

2. User1 goes to connect to HostB. HostA provides the ticket to HostB, which it uses to authenticate User1.

Kerberos includes a user's IP address(es) within authentication tickets to ensure that these tickets cannot be copied and reused by an attacker on another system. This feature of Kerberos prevents SSH man-inthe-middle attacks. However, in environments where network address translation (NAT) firewalls are used, access will be denied when attempting to access a host on the other side of a firewall. In order to enable access across NAT firewalls, Kerberos must be configured to allow tickets that do not include IP addresses, which opens the vulnerability of tickets to being copied and reused.

To facilitate authentication for automated processes, many SSH Kerberos implementations provide the option for credentials to be stored in a file on the client called a keytab file. This enables automated logon without user intervention. Access to keytab files should be restricted to prevent credentials from being compromised and used for unauthorized access from the same system or a different system.

For interactive users, Kerberos is best suited for environments where users require access to several servers, because it provides more secure authentication than password authentication, single sign-on for users, and single point of account management (enabling/disabling, etc.). However, controls must be put in place to limit implicit access and ensure that users only have intended access.

Automated processes should generally have limited, explicit access to a limited number of specific hosts. The exception may be management systems that require automated access to a large number of hosts. In 
practice, Kerberos is rarely used for automated processes and is not recommended due to the risks of implicit access and the lack of command restrictions.

\subsubsection{Public Key Authentication}

Public key authentication in SSH uses user keys or certificates to authenticate a connection - with user keys being the most commonly used method. Such keys can be configured for both interactive users and automated processes, and they authorize the user or process to access a user account in an information system. An interactive user or automated process on an SSH client (HostA) has a user key called an identity key, typically an RSA or DSA private key, and the server (HostB) must have the corresponding public key configured as an authorized key for a user account to which access will be provided. Any user or process in possession of the identity key is then allowed to log into that user account on the server and perform actions under the privileges configured for the account.

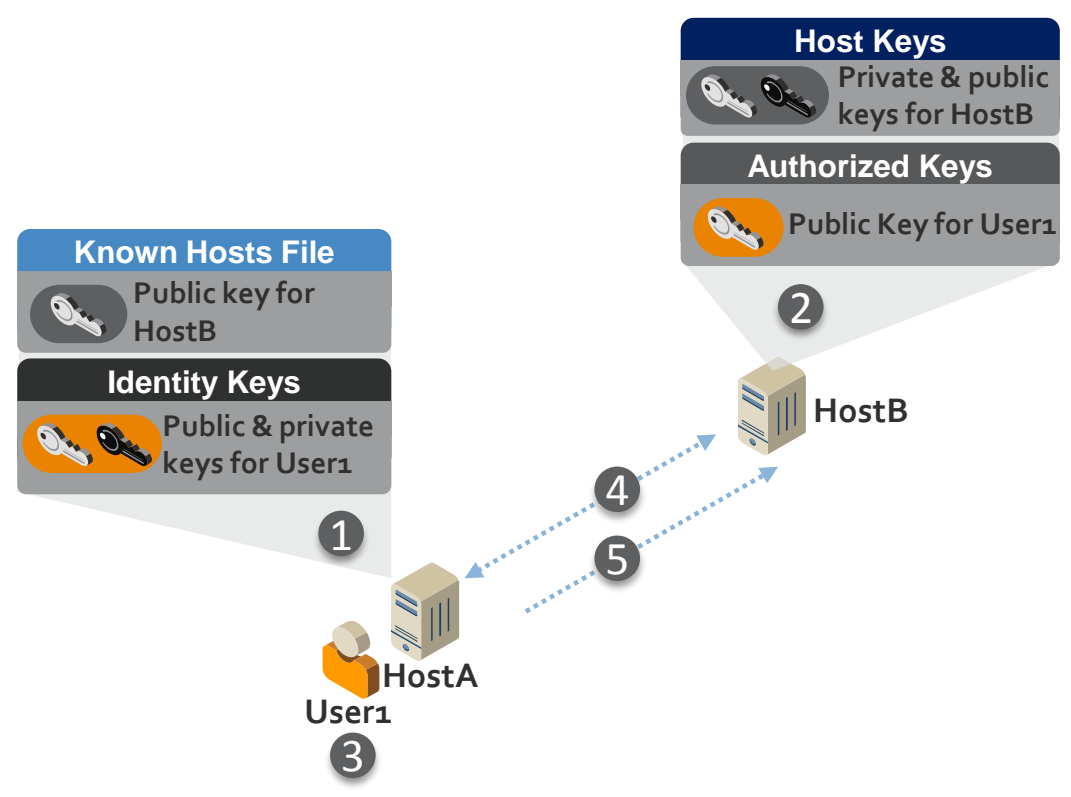

Figure 3-5: Public Key Authentication

Figure 3-5 illustrates SSH public key authentication:

1. User1 or an administrator generates an identity key (and corresponding public key) for User1.

2. The HostB administrator stores User1's public key as an authorized key in the User1 account on HostB.

3. User1 starts to login to HostB.

4. HostA connects to HostB and attempts to authenticate User1 to HostB using User1's identity key.

5. HostB authenticates User1 using the authorized key stored in User1’s account on HostB.

Many SSH implementations support configuring restrictions for authorized keys. These may be used for limiting what can be done on the server using the key (command restrictions) and for limiting the IP addresses from which the key can be used (source restrictions). 


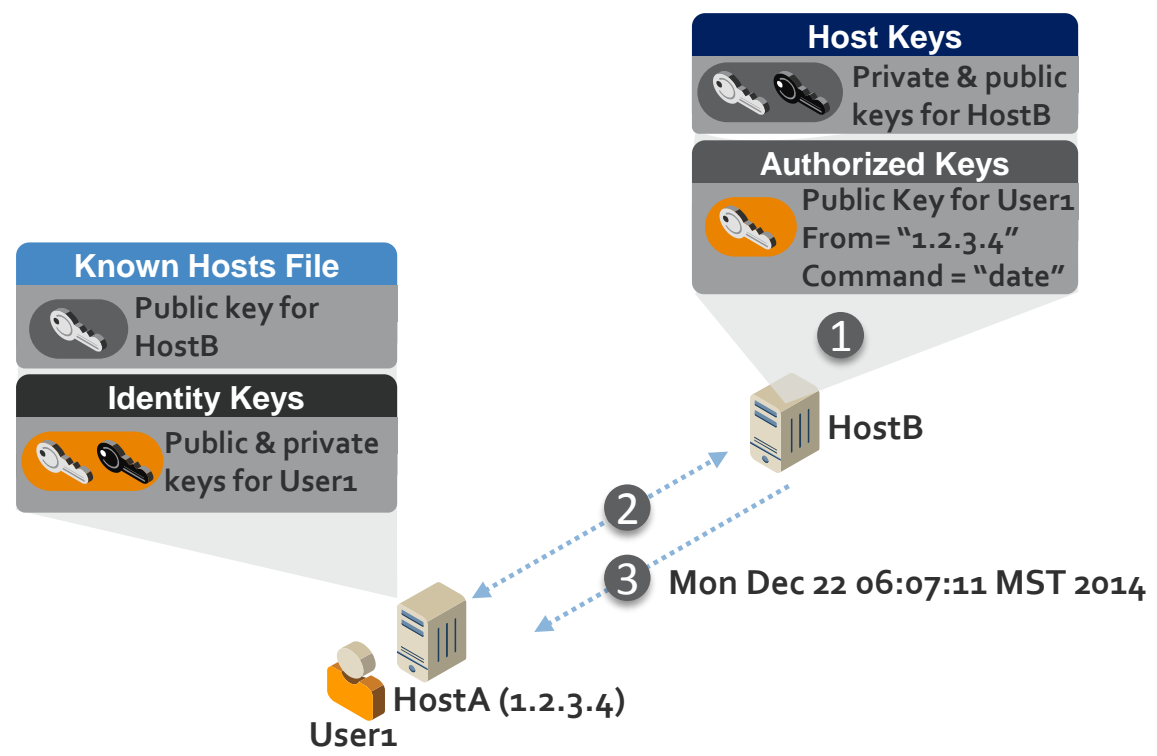

Figure 3-6: Public Key Authentication with Source and Command Restrictions

Figure 3-6 illustrates public key authentication with source and command restrictions:

1. The administrator for HostB configures the authorized key for User1 to only allow authentication to HostB from address 1.2.3.4 and run the date command once authenticated.

2. User1 authenticates to HostB from HostA (IP address 1.2.3.4).

3. HostB executes the date command and then terminates User1's connection.

An important advantage of public key authentication is that it does not create implicit trust relationships, only expressly defined trust relationships, and the permitted access can be reliably determined by inspecting the destination host. ${ }^{6}$ This is very important for being able to clearly determine and audit who can access each system and account. However, if the deployment of authorized keys is not controlled and tracked, users can create unauthorized trust relationships and access that can be exploited.

For interactive users, the identity key is usually stored on a smartcard or in a passphrase-protected file on a client device. If the identity key is protected by a passphrase, it is encrypted by a key derived from the passphrase. When SSH user keys are used for automated access, however, the identity key is usually stored as an unencrypted file (with no passphrase) in the file system. ${ }^{7}$ Given that the files grant access to servers, they contain sensitive data.

Public key authentication is the recommended authentication mechanism for automated access with SSH due to the command and source restrictions that are available and the ability to more readily prevent implicit trust. Public key authentication is by far the most frequently used method of SSH automated access as of this writing.

6 An exception is OpenSSH's proprietary certificate authentication, which does not allow reliably auditing who can access a host by inspecting just that host. There is no hardened Certificate Authority solution for OpenSSH, and most environments have no systematic tracking or directory of issued OpenSSH certificates. Thus if the host trusts an OpenSSH certificate signing key, it is often not possible to reliably determine who is able to access the host.

7 There is no one present to supply the passphrase for decrypting the identity key for automated access by processes. Hardcoding the passphrase in scripts would provide little additional security (see also NIST SP 800-53 IA-5(7)), and obtaining it from a vault in a script would also provide limited additional security and would be quite a maintenance burden. 


\subsubsection{User Authentication Summary}

There are many considerations to take into account when selecting authentication methods for SSH across an enterprise. Table 3-1 summarizes the major security and operational considerations for the user authentication methods described in this section in commonly used SSH implementations. Generally, the goal should be to select a single method for all authentications or one method for all interactive users and one for all automated processes. However, this is not always possible based on the options available in deployed SSH systems and software.

For automated processes, public key authentication is the recommended method based on its additional security features. For interactive users, public key authentication with smartcards provides the best security. For file-based public key authentication with interactive users, if passphrase strength for interactive user identity keys stored in files (instead of smartcards) cannot be enforced, weak passphrases may put identity keys at risk of compromise. In these cases, password or Kerberos authentication, where password strength can be enforced, may provide higher security. The use of keytab files with Kerberos for interactive users is not recommended.

If multiple authentication methods will be enabled, it is critical to put controls in place to prevent unintended access. For example, if Kerberos is selected for interactive users and public key authentication for automated processes, it is critical to ensure that the names of system accounts for automated processes are not the same as accounts in the central directory. And, conversely, it is also critical that users be prevented from configuring authorized keys that would allow them circumvent controls enforced via Kerberos.

Table 3-1: Comparing Authentication Methods

\begin{tabular}{|l|c|c|c|c|}
\hline & Password & Host-Based & Kerberos & Public Key \\
\hline $\begin{array}{l}\text { Supports Portability (User not required to carry } \\
\text { credential from system to system) }\end{array}$ & Yes & Yes & Yes & Yes/No $^{8}$ \\
\hline Provides Single Sign-On & No & No & Yes & Yes \\
\hline Enforces Command Restrictions & No & No & No & Yes \\
\hline Prevents Man-in-the-Middle $^{9}$ & No & Yes & Yes & Yes \\
\hline Minimizes Implicit Access & Yes/No ${ }^{10}$ & Yes & No & Yes \\
\hline Supports NAT Environments & Yes & Yes & No & Yes ${ }^{11}$ \\
\hline
\end{tabular}

8 If the identity key for an interactive user is stored on a smartcard, the user can easily and securely carry it from system to system. If it is stored in a passphrase-protected file, the user must copy the file to each client from which he or she accesses SSH servers.

9 This assumes that best practices are being followed. For example, if the server's public key is not properly validated, a manin-the-middle attack is possible with any authentication method.

10 When password or keyboard-interactive authentication is used with LDAP, implicit access can occur when an account on a host unexpectedly has the same name as an account in the LDAP directory.

11 Source restrictions must be configured to use the address on the server side of the NAT device. 


\section{Vulnerabilities in SSH-Based Access}

Because SSH is the primary secure access method used for administration and automated processes on mission critical systems, its security is crucial. The systems managed via SSH include Unix and Linux systems, routers, firewalls, security appliances, Unix partitions on mainframes, and other network devices. The privileges granted to users and automated processes via SSH are typically elevated—often root level—privileges. ${ }^{12}$ A number of vulnerabilities arise—-both for users and automated processes_-if proper provisioning, termination, and monitoring processes are not implemented.

The security of SSH-based automated access, and even interactive access, has been largely ignored to date. Many organizations don't even know how many SSH keys they have configured to grant access to their information systems or who has copies of those keys. The accounts associated with these keys often grant far more access than is actually needed, such as allowing execution of any command or transfer of files to any directory. Also, in many organizations, system administrators configure new keys without any approvals or coordination, and may use them to circumvent auditing of privileged access and maintenance. Some large enterprises have hundreds of thousands or even millions of SSH user keys on their systems for automated access, which often provide many more entry points onto servers than the interactive user accounts do. Also, a sizable percentage of these keys typically grant access to administrative/root accounts or sensitive accounts, such as those storing database files or critical software.

SSH is also often used for communications with other organizations, external systems, or independent users. A closely related issue is the trust relationships that these keys establish within and between systems, even between organizations. Some of these trust relationships may be undesirable or violate policies, such as leading from development and test systems into production systems, or crossing from a low-impact system to a high-impact system without requiring any additional authentication. Most importantly, SSH key-based trust relationships can enable an attacker who compromises one system to quickly move (or pivot) from one system to another and spread through an organization-individual trust relationships often collectively form webs of trust across an organization's systems.

Although the security implications of poor SSH key management have been known for some time, the scope of the problem for automated access has not been widely understood or acknowledged. For example, most organizations do not have SSH key management or SSH-based automated access as part of their assessment programs, even though for many organizations SSH-based automated access has already become central to their identity and access management operations.

This section describes the primary categories of vulnerabilities in SSH-based interactive and automated access - with a particular focus on user keys used to grant access in public key authentication. The recommendations presented in subsequent sections of this document are intended to address these vulnerabilities. The categories of vulnerabilities described in this section are as follows:

- Vulnerable SSH implementation

- Improperly configured access controls

- Stolen, leaked, derived, and unterminated SSH user keys

- Backdoors (unaudited user keys)

- Unintended usage of user keys

12 Although access to root accounts via SSH is generally not allowed, administrators will leverage sudo and other tools to elevate their privileges for many operations once logged in via SSH. 
Pivoting

Lack of knowledge and human errors

\subsection{Vulnerable SSH Implementation}

An SSH server or client implementation could have vulnerabilities that allow it to be exploited in order to gain unauthorized access to communications or systems. These vulnerabilities could be any of the following types:

- Software flaws in the SSH implementation (i.e., coding errors)

- Configuration weaknesses (for example, allowing the use of weak encryption algorithms)

- Protocol weaknesses (for example, supporting the use of SSH version 1)

\subsection{Improperly Configured Access Controls}

In its role of enabling administration of systems via elevated privileges_including root-SSH is highly susceptible to enabling unauthorized access due to improperly configured access controls. Most SSH implementations provide a broad number of options for configuring and controlling access, some of which are provided by the SSH software and others through components with which the SSH software integrates, including the underlying OS, pluggable authentication module (PAM), Kerberos, and other security components. Although powerful, improperly configured access controls can inadvertently enable SSH access directly to the root account, unauthorized access to other accounts due to implicit access enabled via Kerberos, the ability to elevate privileges by manipulating the environment, and other accessbased vulnerabilities. An administrator may, for example, properly configure SSH but incorrectly configure PAM or not sufficiently limit permissions at the OS for the account being accessed.

Furthermore, each OS, appliance, device, or application that supports SSH features different options and methods for controlling access, further complicating efforts to ensure security.

\subsection{Stolen, Leaked, Derived, and Unterminated Keys}

Stolen, leaked, derived, and unterminated identity keys pose a similar problem to stolen, leaked, derived, and unterminated interactive user account credentials. Anyone who may have obtained a copy of an identity key — by copying it from a host, by accessing a backup, by having malware harvest keys, by performing factoring to derive a key, etc.-may use that key to attempt to gain unauthorized access to a user account on one or more servers in the organization. Once access to a user account has been gained, it is generally possible to access and modify any data for that user account-including reading and modifying the memory of processes running under that user account, and modifying any executable programs owned by that user account.

\subsection{Backdoor Keys}

Many organizations mandate that all privileged access to their servers take place through a privileged access management system that records all actions performed. Unfortunately, SSH public key authentication can be used to create a "backdoor" that bypasses the privileged access management system. This is done as easily as generating a new key pair and adding a new authorized key to an authorized keys file; authorized keys files are often not audited, so an added key may remain unnoticed for years. The corresponding identity key can then be used to log into the server using any SSH client without the privileged access management system recording the ensuing activity. 


\subsection{Unintended Usage}

Users may, intentionally or unintentionally, use identity keys for purposes for which they were not intended. An example is using an identity key that was only intended to be used for automated file transfers to tunnel traffic (thus concealing it from network security controls).

\subsection{Pivoting}

Malware can be engineered to use SSH keys to spread when automated access is allowed. The mesh of interactive and automated access relationships is so dense in many cases that it is likely that an attack can spread (pivot) to most servers in an organization after penetrating the first few servers, especially if other attack vectors are used to escalate privileges. See Figure 4-1 for an illustration of pivoting.

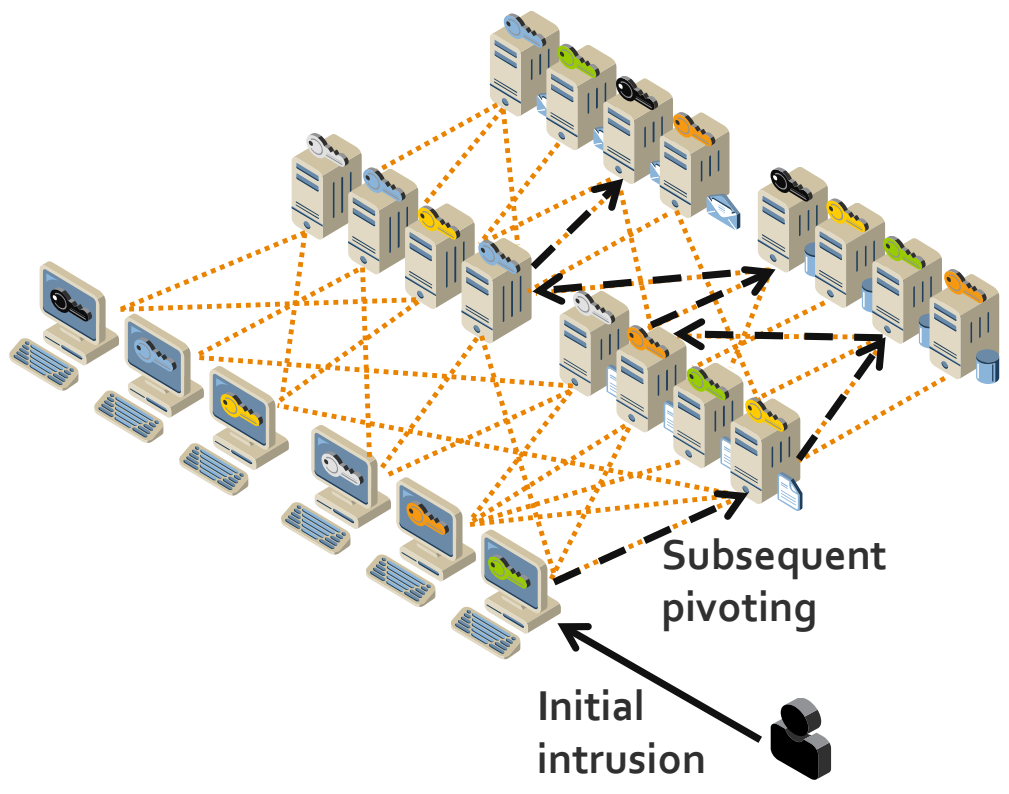

Figure 4-1: Pivoting Enabled by Chained SSH Trust Relationships

\subsection{Lack of Knowledge and Human Errors}

One of the greatest ongoing challenges to the security of SSH-based systems is the potential for human error due to the complexity of SSH management and the lack of knowledge many administrators have regarding secure SSH configuration and management. An authorized key may inadvertently be deployed incorrectly on a host, such as to a root account instead of a regular user account, thus granting unnecessary privileges. People are known to make human errors when manually setting up new trust relationships. Such errors can go undetected for years. Also, some key setups involve thousands of hosts, and while it is easy to miss one or more hosts when copying an authorized key to so many hosts manually, debugging such errors can be very time consuming. Also, when manually fixing problems, administrators are likely to just copy the missing keys to the proper accounts without, for example, checking whether they have accidentally been copied to the root account. 


\section{Recommended Practices for Management}

Effectively securing SSH access consists of defining clear policies and implementing management, operational, and technical security control processes to address already deployed SSH systems, privileges, and user keys, and ensure that new SSH systems and user keys are properly deployed and tracked. The objective of management is to improve security as economically as possible by following the NIST SP $800-53^{13}$ recommended practices for access control with respect to interactive and automated access using $\mathrm{SSH}$.

Operational processes can be optimized by automating SSH user key setups and removals and related approval, documentation, monitoring, and audit processes. In particular, it is possible to integrate a key management system with a ticketing or change tracking system. If requests for provisioning interactive or automated access are made using a predefined template, a key management system can automatically do the provisioning once the request has been approved, thus reducing labor. Certain provisioning requests may touch thousands of hosts, and some organizations are known to use person-years annually on manual provisioning.

An SSH access management project may also include either selection and acquisition of software tools to aid in the process or development of automatically or manually executed scripts.

\subsection{SSH Security Policies and Procedures}

Policies and procedures play a critical role in SSH security by establishing consistent baseline requirements across the diverse systems and environments where SSH is deployed, including rapidly evolving cloud environments. The definition of policies should clearly spell out roles and responsibilities in order to prevent misunderstandings that result in security lapses and to ensure accountability. It is critical to educate all SSH stakeholders (system administrators, security professionals, business application owners, etc.) on SSH security policies and processes.

\subsubsection{Secure SSH Implementation}

Although the hardening of SSH implementations is outside the scope of this document, it is worth noting a few areas where policies and procedures should be defined to mitigate the significant vulnerabilities that can emerge due to poor implementation and management. Other NIST publications and industry standards should be consulted as references for defining policies in the following areas:

- Only enabling SSH server functionality on systems where it is absolutely required.

- Keeping SSH server and client implementations fully up to date across all systems.

- Hardening SSH server and client implementations, including disabling SSH v1 protocol, disabling unapproved authentication methods, preventing implicit access by limiting SSH accessible accounts and groups (including root), disabling port forwarding, limiting access to environment variables, using approved ciphers, properly configuring supporting subsystems (e.g., PAM), and enforcing SSH inactivity timeouts. ${ }^{14}$

\section{http://dx.doi.org/10.6028/NIST.SP.800-53r4}

14 Unfortunately, it is not always possible to harden SSH implementations (e.g., on appliances and embedded devices). In these cases, compensating controls can be used to mitigate the vulnerabilities, such as implementing virtual private network (VPN) tunnels to encapsulate the potentially vulnerable SSH traffic. 
Enforcing least privileged access for all SSH-accessible accounts and groups, especially for automated processes and remote access.

\subsubsection{SSH Identity and Authorized Keys}

SSH user keys are security-sensitive configuration information for SSH clients and servers, and their misconfiguration, improper disclosure, or modification may expose servers, applications, and information to unintended access or vulnerabilities. The following policies and procedures are recommended:

Minimum Key Lengths and Approved Algorithms: To prevent factoring by an attacker, user keys must conform to organizational standards for minimum key lengths used with approved algorithms. NIST SP $800-131 \mathrm{~A}^{15}$ currently provides recommendations for minimum key lengths that are considered secure in conjunction with various algorithms. Updates to NIST SP 800-131A should be monitored to ensure that the latest recommendations for key lengths are being followed. Note that some older SSH implementations may not support the key lengths recommended by NIST SP 800131A. Clear criteria should be defined for exceptions where the use of smaller key lengths is allowed.

- Cryptoperiods (Maximum Key Lifetime): To minimize the risk of key compromise due to administrative turnover or improper key management by users, the maximum time (cryptoperiod) that an identity key may be used before replacement with a new key should be specified. NIST SP 800$57^{16}$ provides recommended cryptoperiods for public/private authentication keys. Cryptoperiod policies may take into account the potential for compromise based on types of use, including identity keys used for users versus automated processes, the criticality of systems that are being accessed, the environment where identity keys are stored (e.g., mobile laptops versus smartcard), and whether the keys are used in connections that span outside the organization.

- Identity Key Access Control: To prevent compromise of a key by an authorized user, access controls on identity keys should be configured to restrict access to the interactive user or automated process to which they have been assigned. For automated processes, policies should define guidelines for assigning access to administrative staff responsible for managing identity keys.

- Identity Key Passphrases: To protect against compromise, identity keys assigned to interactive users should be protected by a passphrase. Standards for passphrase strength and changing passphrases each time a key is replaced (at the end of the cryptoperiod) should be defined.

- Identity Key Duplication: Identity keys should not be duplicated (copied to other systems) for interactive users and automated processes. If duplication is allowed for interactive users, guidelines should be provided for the acceptable locations where keys can be copied and used.

- Authorized Key Access Controls: Access controls on authorized keys should ensure that nonsuperusers cannot install new authorized keys for user accounts they use. Such new authorized keys can create backdoors, bypass privileged access auditing systems, grant permanent access, or grant access to others, without regards to controls on non-local access and authorization boundaries. In practice this means "locking down keys", i.e., moving authorized keys to superuser-owned locations that are not writable to non-superusers. It is strongly recommended that authorized keys be moved to protected locations where ordinary users cannot add new or change existing keys, as well as configuring SSH servers to only look for keys from those locations. This is necessary for the following reasons:

o Maintaining control of who can access what information

15 http://csrc.nist.gov/publications/nistpubs/800-131A/sp800-131A.pdf

16 http://csrc.nist.gov/publications/PubsSPs.html 
o Properly identifying users accessing the system

o Preventing a user from accessing the system after his/her account is terminated

o Ensuring that users cannot bypass privileged access systems

o Controlling remote access

o Enforcing authorization boundaries

- Authorized Key Command Restrictions: Authorized keys used for automated processes should be configured with command restrictions unless special approval is given. Command restrictions are particularly important for keys used for authorizing remote file transfers to avoid accidentally permitting remote terminal access and remote execution of commands. For interactive users, command restrictions used in conjunction with scripts may be used to limit the commands available to users.

- Authorized Key Source Restrictions: Authorized keys used for automated processes should be configured with source restrictions unless special approval is given, i.e., restrictions on the client IP addresses from which the keys can be used. This prevents an attacker who compromises an identity key from using it elsewhere on the network. Source and command restrictions are a particularly important consideration for SSH connections that allow access from outside the organization.

- Replacement on Compromise or Reassignment: SSH keys should be changed when a compromise is suspected. In addition, identity keys used for automated processes should be changed prior to cryptoperiod expiration whenever an administrator responsible for managing the keys for one or more automated processes has been reassigned or terminated.

- Usage Logging: All SSH servers should be configured to log key fingerprints for access based on SSH authorized keys. This is necessary for performing the log analysis needed for continuous monitoring and forensic investigations.

- Pivot Prevention: Accounts should not be configured with both incoming and outgoing identity keybased trust relationships unless expressly needed (for example, an approved jump server). For example, if an authorized key for IdentityKey1 is configured for account User1 on Server1 and that same account has another identity key enabling it to authenticate to account User1 on Server2, an attacker who gains access to IdentityKey1 can now pivot from Server1 to Server2. Incoming and outgoing trust relationships on a server should be configured in separate accounts to prevent pivoting. If an organization has needs for limited pivoting, these implementations should be approved on a case-by-case basis.

- No Environment Crossing: SSH trust relationships should not cross release environment (dev, QA, prod) in order to prevent a compromise in a "lower" development or QA environment spreading to production environments.

\subsection{SSH Key-Based Access Provisioning, Life Cycle, and Termination Processes}

Provisioning and configuring access to an account for interactive users and automated processes should be a judged decision, balancing the need for access against the risks, and should include consideration of the level of access required. It is not acceptable for any user or system administrator to grant user account access to other users or processes without proper approval. 
In a controlled provisioning and life cycle process, provisioning interactive or automated access requires the phases depicted in Figure 5-1:

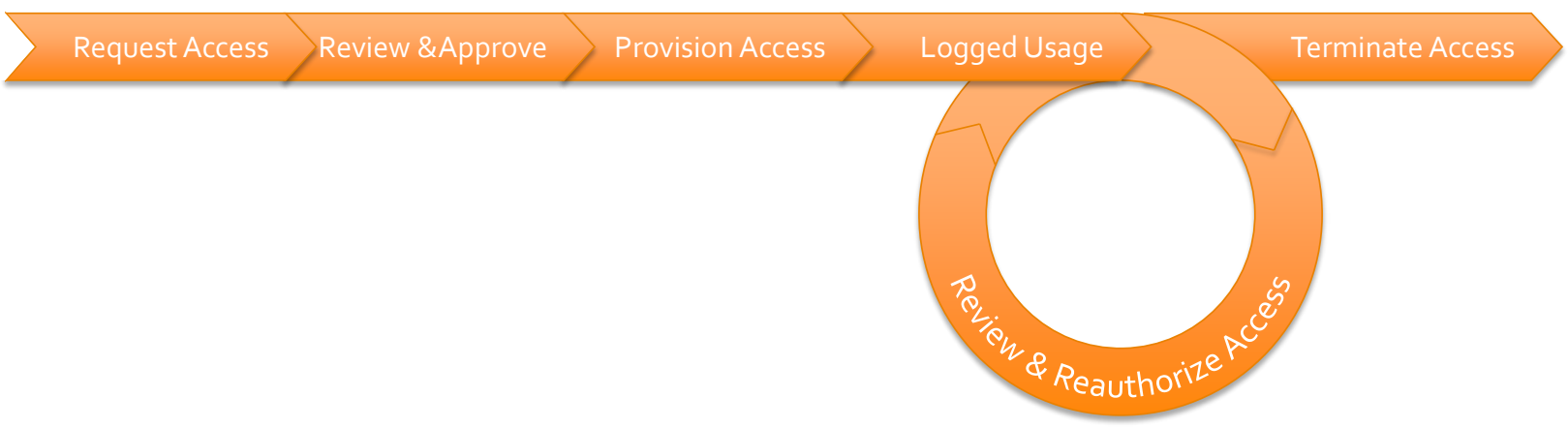

Figure 5-1: Controlled Provisioning and Life Cycle Process

- Request: Submitting a request for establishing access to one or more accounts on one or more servers together with justification for the access. The request for automated access should specify the command that is authorized to be executed (or in exceptional cases, interactive command line access). Furthermore, a request should identify the business process, application, or information system that the request relates to and the owner/responsible person for the trust relationship if not the owner of the business process, application, or information system. The request should also specify the source account(s) and host(s) from which access is allowed or the key to be used for access. Ideally, an existing change control system is used for submitting a filled request template.

- Approval: Reviewing and approving the request based on consideration of security impact, destination account and server, authorization boundary crossing, and remote access considerations. Ideally, the change control system is used for approving the change in large enterprises or other organizations that need centralized, automated mechanisms for managing keys. The request should, at a minimum, be reviewed and approved by the official responsible for the security of the information system to which it grants access. Access grants create connections between systems and may need to be documented in security policies, plans, or architecture documents as system interconnections. Access grant approvals should be recorded in a protected database to enable them to be used for continuous monitoring and audits. A controlled process with proper documentation of approvals is necessary for being able to detect access grants by unauthorized users (e.g., unapproved authorized keys) during continuous monitoring. Without documented approvals, it will not be possible to audit that all existing authorized keys have been approved. Documenting why each key is authorized is also important for being able to remove keys when no longer needed to properly terminate access.

- Provisioning: Implementing the request, creating a key pair if needed, and configuring the authorized key(s) and identity key(s). SSH key-based access provisioning should be limited to authorized individuals; ideally, it is performed by a central team that ensures consistent management across all systems. To improve efficiency and reduce errors, the implementation takes place automatically to reduce resources, reduce the need for privileged access, and eliminate manual configuration errors.

- Usage Logging: Logging usage to facilitate reauthorizations (e.g., accounts and authorized keys that are not used for a long period of time should likely not be reauthorized), forensic investigations, and audits. Logs should be retained for a sufficient amount of time to enable forensic or operational investigations at a later date. Log files should be safeguarded because they may contain sensitive 
information. For more information on logging, see NIST SP 800-92, Guide to Computer Security Log Management. ${ }^{17}$

- Review and Reauthorization: Regular reviews and reauthorization of SSH key-based access by individuals responsible for the security of systems and data being accessed. An organization should define policy on how often approvals need to be revalidated for each information system, taking into account the impact level of the system and the level of access granted (e.g., whether the access is to a privileged account, whether execution of arbitrary commands is permitted). Ideally, in a centrally managed system the revalidation process should be continuously monitored and reflect the immediate need for individuals and systems to have access to the required resources. To facilitate a holistic review of access to servers and applications, the review of entitlements granted via authorized keybased trust relationships should be integrated with existing privileged access management reviews.

- Termination: Terminating access for automated processes when an application is decommissioned or the automated trust relationship is no longer required by the application. Access for interactive users should be terminated when a person leaves the organization or changes roles.

\subsection{Establish Continuous Monitoring and Audit Processes}

The purpose of continuous monitoring is to ensure that the processes for provisioning, life cycle management, and termination are followed and enforced. It is also important for detecting unauthorized access that may have been illicitly provisioned by attackers as a backdoor into systems. Unauthorized and misconfigured SSH user keys should be detected. For example, anyone or anything (including malware) with access to a superuser account can technically configure new authenticators granting access to any account.

One approach is to periodically discover all configured authorized keys for all user accounts on all servers, check for each authorized key whether there is a corresponding valid approval (and that the key has the same access restrictions, such as command restriction, as in the approval), and generate alerts whenever the configured access does not have a matching approval. In other words, this involves establishing a baseline and whitelisting that baseline to detect any deviations from the accepted baseline. Ideally, SSH client configurations are also analyzed, and any configured identity keys are checked against approvals to ensure that the private key corresponding to each approved authorization is not found from any unauthorized locations.

Log data generated by an information system should be analyzed to 1) detect configured SSH user keys that are not being used and propose them for removal to eliminate unnecessary access grants (many of these keys likely belong to users who have left the organization); 2) detect and monitor connections from remote locations and connections crossing authorization boundaries and ensure that they are properly approved; 3) detect connections using an authorized key from hosts from which connections have not been authorized (such connections would typically involve leaked credentials and warrant immediate key rotation); and 4) identify keys that are used from outside the managed environment as “external keys" requiring manual rotation. Ideally, such log data analysis integrates with an existing SIEM or other log data collection system used by the organization.

Furthermore, continuous monitoring should ensure that remote access and non-local maintenance (including privileged access) using SSH user keys is properly audited, and that SSH/SFTP file transfers using SSH user keys and crossing a security zone boundary are properly audited and content-checked and

17 http://csrc.nist.gov/publications/PubsSPs.html\#800-92 
are not used for unintended purposes (such as executing commands or transferring files in the wrong direction).

Auditing of SSH user keys serves risk analysis and ensures that the provisioning, life cycle management, and termination processes as well as continuous monitoring are working properly. A comprehensive audit of SSH user keys for risk analysis purposes should be performed by all organizations that use SSH or SFTP protocols (including within file transfer products or for network device or hardware/BIOS-level configuration). Audits for ensuring the functioning of processes, on the other hand, can use representative sampling and condition detection tests to gain sufficient confidence that the processes are functioning.

\subsection{Inventory and Remediate Existing SSH Servers, Keys, and Trust Relationships}

Many organizations have thousands of untracked identity keys, authorized keys, and corresponding trust relationships granting access across a large number of mission critical systems. Existing legacy keys pose a substantial security risk and make risk analysis difficult if they are not understood. An inventory of the location of all existing SSH keys and an inventory of trust relationships must be created and evaluated against defined policies. The inventory should include the locations (host and account) of all identity and authorized keys and authorized key restrictions (e.g., source and command restrictions).

Remediation is also necessary for compliance. NIST SP 800-53 requires identifying authorized users and their access rights, understanding and properly authorizing connections between information systems, applying the principle of least privilege, and managing authorized keys, among other requirements.

An inventory and remediation project typically consists of:

- Mapping all trust relationships that result from deployed identity and authorized keys and requiring review and approval from their owners (including description of the purpose of the key or other justification for the existence of the key). Trust relationships created by public key authentication must be reviewed and evaluated based on the potential for chains of trust relationships coming in and out of systems to be used to pivot. Source and command restriction information should be included to enable evaluation of proper access controls and restrictions. Any keys for which such approval is not deemed appropriate (such keys may be backdoors and may have been left behind by attackers) should be removed. Ideally, trust relationship information can be imported into existing privilege access management systems for review through standard processes.

- Adding command and source restrictions to authorized keys wherever possible

- Identifying and removing any orphan authorized keys for which a corresponding identity key has not been found, as they may represent backdoor keys which can be used for nefarious purposes

- Identifying and remediating any duplicated identity keys

- Ensuring all identity keys used by interactive users are protected with a passphrase

- Replacing all keys that do not comply with key length and algorithm policies

- Enforcing the SSH identity key provisioning process

- Configuring SSH servers to log fingerprints 
- Locking down keys by moving them to root-owned locations (this is needed to produce a stable state for the later steps)

- Assigning owners (e.g., application, business process owner) for all keys granting access to servers (e.g., based on the server and account they grant access to, possibly automatically using information from a suitable configuration database)

- Monitoring log data for several months to determine which keys are not being used, proposing such keys for removal to their owners, and removing any unused keys that are not specifically approved for keeping (the monitoring period should usually be 6 to 12 months to ensure all active keys are used; the monitoring period should include testing of disaster recovery systems and other processes that may utilize keys)

- Analyzing which keys grant access across authorization boundaries or other defined boundaries (e.g., development/test to production systems, between information systems, remote access) and either categorically removing authorized keys granting such access or obtaining required approvals for such access

- Analyzing which keys are external keys, i.e., used with hosts outside the managed environment

- Rotating (i.e., changing) all identity keys and corresponding public keys (external keys may need manual handling and coordination with administrators of the external hosts with which they are used)

- Onboarding all relevant hosts into a system for managing SSH keys

During the remediation project, relevant SSH configurations should be backed up before making a change so that the change can be quickly rolled back in case something breaks.

A key remediation project can be a sizable effort, but it is critical for security and compliance. Note that it is generally considered infeasible to maintain a $100 \%$ inventory of all SSH identity keys because they are such small files that can be stored anywhere, even on paper (they are small enough to be typed in). The real goal is to keep an inventory of all authorized keys and to the extent possible, the corresponding identity keys.

\subsection{Automate Processes}

The automation of the processes involved in the management of SSH key-based access can significantly improve security, efficiency, and availability. This includes the following processes:

- Inventory: Manual discovery and inventory of all SSH identity and authorized keys (along with corresponding restrictions) and mapping of all resulting trust relationships is practically impossible. To accurately complete an SSH key inventory across an enterprise, or even a single department, automation of some sort is essentially a requirement. Initial identification of all SSH servers to start the process of key discoveries can be performed by leveraging existing vulnerability scanning systems.

- SSH Key-Based Access Review: Automatically importing trust relationship data into an existing privilege access management system for review leverages existing processes and facilitates an accurate review and tracking of approvals for access granted through SSH keys. 
- Provisioning: System administrators in many organizations spend a substantial amount of time configuring and managing SSH user keys. Many organizations already use a ticketing or change control system for approving changes to their IT systems. Provisioning interactive or automated access using SSH is most naturally performed using the same system used for approving other IT changes or other access. Ideally, a special template is used for filling a request for interactive or automated access, and once approved, the request is automatically picked up by a key management system and implemented on all affected hosts. Such automation eliminates the manual steps for setting up keys, eliminates the need for manual root access for installing the keys, reduces the amount of privileged administrative access that needs to be audited and reviewed, eliminates configuration errors due to incorrectly implemented requests, and ensures that the approval template remains available for future reference and for use in continuous monitoring and audits.

- Continuous Monitoring: Automation is also a virtual necessity for any continuous monitoring process.

- Audit: A system that can provide central reporting and analysis tools across the inventory can reduce the time necessary to prepare the data required for audits and make it easier for auditors to verify proper implementation and identify exceptions.

\subsection{Educate Executive Management}

Many executives are not aware of the central role SSH keys play in the secure management and operation of mission critical infrastructure and the significant breaches that can occur if they are exploited. Without sufficient executive education for both security and operationally focused executives, SSH key management initiatives can get derailed by other seemingly higher priorities, leaving an organization vulnerable. To ensure the proper level of priority is placed on the implementing and sustaining SSH key management, it is important that executives understand the systems on which SSH is used, the levels of access granted to users and automated processes via SSH, the risks and potential impacts of an SSH-based breach (including pivoting), and the basic steps needed to implement an SSH key management program (as outlined in this document). 


\section{SSH-Based Access Management Planning and Implementation}

This section discusses considerations for planning and implementing the management of SSH keys for interactive and automated access. It assumes that the organization's processes involving SSH-based interactive and automated access management are primarily ad hoc-lacking policies and requirements, lacking standardized processes and automated tools for key management, etc. As with any technology deployment, planning and implementation of SSH key management for interactive users and automated processes should be addressed in a phased approach. A successful deployment can be achieved by following a clear, step-by-step planning and implementation process. The use of a phased approach for deployment can minimize unforeseen issues and identify potential pitfalls early in the process. This model also allows for incorporating advances in new technology and adapting the technology to the everchanging enterprise. The following is an example of planning and implementation phases:

1. Identify Needs. The first phase involves identifying the needs to manage SSH keys for interactive and automated access and determining how those needs can best be met.

2. Design the Solution. The second phase involves all facets of designing the solution. Examples include cryptographic settings, key management, and management automation.

3. Implement and Test a Prototype. The next phase involves implementing and testing a prototype of the designed management solution in a lab or test environment. The primary goals of the testing are to evaluate the functionality, performance, scalability, and security of the management solution, and to identify any issues with the components, such as interoperability issues.

4. Deploy the Solution. Once the testing is completed and all issues are resolved, the next phase includes the gradual deployment of the management solution throughout the enterprise.

5. Manage the Solution. After the management solution has been deployed, it is managed throughout its lifecycle. Management includes maintenance of its components and support for operational issues. The lifecycle process is repeated when enhancements or significant changes need to be incorporated into the solution.

This document does not describe the planning and implementation process in depth because the same basic steps are performed for any security management technology. This section only highlights those considerations that are particular to managing SSH keys for interactive or automated access and were not already discussed in previous sections of this document.

Organizations may follow a project management methodology or life cycle model that does not directly map to the phases in the model presented here, but the types of tasks in the methodology and their sequencing are probably similar.

\subsection{Identify Needs}

The purpose of this phase is to identify the needs to manage SSH-based interactive and automated access and determine how those needs can best be met through use of automated enterprise management tools, ad hoc implementation of a key management system, etc. Requirements specific to identifying needs that should be considered include the following:

- Engage Stakeholders. Because SSH use spans so many technologies and groups, it is important to identify and engage stakeholders, including groups with large numbers of SSH-based systems (e.g., 
Unix and Linux teams, applications, groups leveraging cloud services), auditors, and executive management to understand needs and requirements.

- Current Management Processes. A good understanding of existing SSH key management processes, including any existing automated management tools or scripts is helpful.

- Existing Requirements. There may already be mandates, regulations, organization policies, etc. that prescribe requirements for managing SSH keys used for interactive and automated access.

- Volume of Activity. This should include the approximate number of devices and users/processes currently using SSH-based interactive and automated access, and the approximate number of additional devices and users/processes that could benefit from SSH-based access.

- System and Network Environments. It is important to understand the characteristics of the organization's system and network environments so that a management solution can be selected that will be compatible with them. Aspects to consider include the following:

o The characteristics of the devices that act as servers, clients, and management administrators, especially the OSs and SSH application versions (servers or clients) they use and their security postures (low-impact vs. moderate-impact, etc.)

o The technical attributes of the interfaces of other systems with which the SSH key management solution might be integrated, such as centralized logging servers, ticketing systems, and security information and event management (SIEM) software

The outcome of the analysis is documentation of the requirements for the SSH key management solutions, including security capabilities (e.g., cryptography, key management), performance requirements, management requirements (including reliability, interoperability, scalability), the security of the technology itself, usability (by both administrators and users), and maintenance requirements (such as applying updates). These requirements will be used to design and test the solution.

\subsection{Design the Solution}

The next phase is to design an SSH key management solution that meets the identified needs and requirements. If these design decisions are incorrect, then the interactive and automated SSH access implementation will be more susceptible to compromise. Major aspects of solution design that are particularly important for SSH-based access management and have not already been covered in this publication are as follows:

- Support for policies and procedures. The solution must support the implementation, tracking, and enforcement of defined SSH key management policies and procedures. The solution must also support the establishment and enforcement of roles and responsibilities through access controls and workflows.

- Cryptographic key management and protection. Key management and protection is another important component of solution design, including key generation, use, storage, recovery, and destruction. ${ }^{18}$ Organizations also need to ensure that access to keys is properly restricted.

18 NIST SP 800-57, Recommendation for Key Management, provides detailed information on key management planning, algorithm selection and appropriate key sizes, cryptographic policy, and cryptographic module selection. This three-part publication is available at http://csrc.nist.gov/publications/PubsSPs.html. 
- Management automation. The sheer number of SSH keys and complexity of resulting trust relationships in most organizations makes effective management via manual processes nearly impossible. As much of the management as possible should be automated, so as to maximize security and minimize human error and resource expenditures. The areas to target for automation include:

o Discovery and inventory of all identity and authorized keys and corresponding configuration data (e.g., source and command restrictions). Tracking of "owners" responsible for management and review of assets within the inventory.

o Trust relationship mapping and integration with privileged access management systems/processes for consolidated and regular review of granted access

o Provisioning and removal of keys, including integration with identity management and other systems to automatically terminate access (remove authorized keys) on change of role or termination

o Continuous monitoring of deployed keys and configuration and validation based on approvals, including the option to automatically remediate unauthorized changes ${ }^{19}$

o Notification of responsible parties on notable events (e.g., potential rogue keys, unauthorized key changes, key expiration, weak key lengths, required access reviews)

o Reporting and dashboard views to enable ongoing analysis and identification of potential anomalies and vulnerabilities not caught by automated monitoring processes

o Audit, including automated collection and consolidation of data required for review

Automating these management functions may involve acquisition of tools and/or development of scripts. The security of all automation components must be carefully reviewed since, by definition, they will operate with administrative-level privileges.

- Integration with existing systems. The management solution must be able to integrate with existing systems and infrastructure, including ticketing systems, SIEM systems, configuration management databases (CMDBs), and privileged access management systems. The solution must address the management of SSH identity and authorized keys across all technologies where they are used-either via automated or manual processes.

\subsection{Implement and Test Prototype}

After the management solution has been designed, the next step is to implement and test a prototype of the design. Ideally, implementation and testing should first be performed on lab or test devices. Only implementations in final testing should be conducted on production devices. Aspects of the solution to evaluate include the following:

- Key Management. Each key should be properly generated, deployed, protected, rotated, and terminated. Each key should only grant the necessary access to authorized resources for authorized users/devices.

- Administration. Administrators should be able to configure and manage all components of the solution effectively and securely. It is particularly important to evaluate the ease of deployment and configuration, including how easily the solution can be managed as the solution is scaled to larger

19 Note that automatic remediation may inadvertently disable critical SSH-enabled capabilities, so it should be used with caution, if at all. 
deployments. Another concern is the ability of administrators to disable configuration options so that users cannot circumvent the intended security. Management concerns should include the effects of changing software settings (e.g., changing cryptographic algorithms or key sizes.)

- Logging and Auditing. Logging and auditing for the management solution should function properly in accordance with the organization's policies and strategies.

- Security of the Implementation. The management solution itself may contain vulnerabilities and weaknesses that attackers could exploit. Organizations should perform extensive vulnerability assessments against the management solution components because of their high value. Another common security concern is the security of the cryptographic keys.

Organizations should consider implementing the components in a test environment first, instead of a production environment, to reduce the likelihood of implementation problems disrupting the production environment. When the components are being deployed into production, organizations should initially use the management solution for a small number of hosts. Many of the problems that occur are likely to occur on multiple hosts, so it is helpful to identify such problems either during the testing process or when deploying the first hosts, so that those problems can be addressed before widespread deployment. A phased deployment is also helpful in identifying potential problems with scalability.

\subsection{Deploy the Solution}

Once testing is complete and any issues have been resolved, the next phase of the planning and implementation model involves deploying the solution. A prudent strategy is to gradually migrate devices and users/processes to the new solution. The phased deployment provides administrators an opportunity to evaluate the impact of the solution and resolve issues prior to enterprise-wide deployment. It also provides time for the IT staff (e.g., system administrators, help desk) and users to be trained.

Most of the issues that can occur during deployment are the same types of issues that occur during any large IT deployment.

\subsection{Manage the Solution}

The last phase of the planning and implementation model is the longest lasting. Managing the solution involves operating the deployed solution and maintaining the policies, software, and other solution components. Examples of typical actions are as follows:

- Testing and applying patches to solution components

- Including additional types of server and client devices in the access management solution

- Performing key management duties (e.g., issuing new keys, revoking keys for compromised systems or departing users)

- Adapting the policies as requirements change. An example is switching to a stronger encryption algorithm or increasing the key size.

- Monitoring the access management components for operational and security issues

- Periodically performing testing to verify that access management is functioning properly 
- Performing regular vulnerability assessments

- Preparing devices for retirement or disposal. Devices and media that hold private keys should be sanitized or destroyed, unless the keys have been retired/rotated. ${ }^{20}$

20 For more information on media sanitization, see NIST SP 800-88 Revision 1, Guidelines for Media Sanitization (http://dx.doi.org/10.6028/NIST.SP.800-88r1). 


\section{Solution Planning and Deployment}

Earlier sections have provided an introduction to authentication methods in SSH with a view towards automated access, described threats and risks associated with poorly managed SSH keys, and provided guidelines on how SSH keys should be managed. This section strives to answer the question, how should one practically proceed to address SSH key management issues?

A typical SSH access management or key management project involves:

- Understanding the current situation, as most organizations have a substantial installed base of SSH keys that have not previously been managed

- Procuring and deploying tools and establishing processes for efficient, controlled provisioning of automated SSH-based access and enforcing approvals according to policy

- Remediating the existing environment, including establishing purpose for every configured authorized key, removing any authorized keys and identity keys that are not used or for which no justifiable purpose can be identified, configuring command restrictions, and rotating any remaining user keys.

An understanding of the existing environment can be obtained through audits. Tools are available that can help in the audit (see Appendix C for a list of tool selection criteria).

Deploying the tools and establishing provisioning processes typically includes locking down keys and integrating into existing IT change control or approval systems. (The integration may be performed at a later stage, or in parallel with the remediation phase.)

In a typical remediation process, each host is taken under management using the tools, each host is monitored for a period of time (preferably several months) to identify which keys are actually used and how, unused/unneeded/rogue keys are removed, trust relationships that cross boundaries inappropriately or violate policy are removed, command restrictions (and source restrictions, if applicable) are added, and all keys are rotated. The remediation process is fairly labor-intensive. Depending on how many keys there are, how readily the owner and purpose of each key can be identified, and how much work is needed for writing change requests and waiting for maintenance windows, remediating a host may take several hours of work (tools can help a lot, but they cannot determine the purpose or owner of each key if that information hasn't been recorded anywhere).

Generally it has been found that the cost of manual labor in a key management project is often as big or even significantly bigger than the cost of the tools for managing the keys. The choice of tools has a major impact on the amount of manual labor needed. 


\section{Appendix A-NIST SP 800-53 Controls Mapping}

This appendix lists the NIST SP 800-53 Revision $4^{21}$ security controls that are most pertinent for securing SSH-based automated access management. Next to each control is an explanation of its implications particular to SSH-based automated access management.

\begin{tabular}{|c|c|}
\hline NIST SP $800-53$ Controls & SSH Implications \\
\hline $\begin{array}{l}\text { AC-2, ACCOUNT MANAGEMENT, } \\
\text { control \#d }\end{array}$ & $\begin{array}{l}\text { SSH user keys authorize access to the information system and specify } \\
\text { privileges for access. }\end{array}$ \\
\hline $\begin{array}{l}\text { AC-2, ACCOUNT MANAGEMENT, } \\
\text { control } \# g\end{array}$ & $\begin{array}{l}\text { Enhanced auditing of SSH should be enabled to track the usage of keys } \\
\text { and provide an audit trail of which source user (and client) is using keys to } \\
\text { connect to the destination user. }\end{array}$ \\
\hline $\begin{array}{l}\text { AC-2, ACCOUNT MANAGEMENT, } \\
\text { control \#i }\end{array}$ & $\begin{array}{l}\text { Valid authorization should be required before installing an SSH authorized } \\
\text { key, because such keys grant access to the system. Individual users or } \\
\text { administrators should not be able to grant access to friends or colleagues } \\
\text { without control. Granted key-based access should be limited to intended } \\
\text { usage (e.g., intended command). }\end{array}$ \\
\hline $\begin{array}{l}\text { AC-2, ACCOUNT MANAGEMENT, } \\
\text { control \#j }\end{array}$ & $\begin{array}{l}\text { SSH keys should be monitored periodically for compliance with key } \\
\text { management policies. }\end{array}$ \\
\hline $\begin{array}{l}\text { AC-2, ACCOUNT MANAGEMENT, } \\
\text { control \#k }\end{array}$ & $\begin{array}{l}\text { Any private keys held by a group of individuals should be rotated } \\
\text { whenever an individual is removed from the group (note: administrators } \\
\text { may obtain copies of keys when using service accounts). Keys stored on } \\
\text { shared accounts on jump servers should be rotated when someone's } \\
\text { access to the jump server is terminated. }\end{array}$ \\
\hline AC-3, ACCESS ENFORCEMENT & $\begin{array}{l}\text { Approvals for key-based access should be enforced. Users should not be } \\
\text { able to install unapproved keys (keys must be locked down). }\end{array}$ \\
\hline $\begin{array}{l}\text { AC-3, ACCESS ENFORCEMENT, } \\
\text { control enhancement \#3 }\end{array}$ & $\begin{array}{l}\text { Users should be prevented from propagating their access rights by } \\
\text { installing new private keys. }\end{array}$ \\
\hline $\begin{array}{l}\text { AC-4, INFORMATION FLOW } \\
\text { ENFORCEMENT }\end{array}$ & $\begin{array}{l}\text { SSH keys should be managed in order to have control over the flow of } \\
\text { information between interconnected systems. }\end{array}$ \\
\hline $\begin{array}{l}\text { AC-4, INFORMATION FLOW } \\
\text { ENFORCEMENT, control enhancement } \\
\# 4\end{array}$ & $\begin{array}{l}\text { Content of encrypted SSH sessions and SFTP file transfers should be } \\
\text { content-checked (e.g., run through data loss prevention (DLP) software, } \\
\text { antivirus software). }\end{array}$ \\
\hline AC-6, LEAST PRIVILEGE & $\begin{array}{l}\text { Command restrictions should be configured for SSH authorized keys } \\
\text { whenever possible to limit what can be done with the keys. Key-based } \\
\text { access to root accounts should be limited to cases where it is necessary } \\
\text { to accomplish the task. Only those authorized keys that are necessary for } \\
\text { accomplishing the assigned tasks should be configured. In corollary, it is } \\
\text { necessary to know what task each key relates to. }\end{array}$ \\
\hline $\begin{array}{l}\text { AC-6, LEAST PRIVILEGE, control } \\
\text { enhancement } \# 2\end{array}$ & $\begin{array}{l}\text { SSH authorized keys for privileged accounts should only be configured if } \\
\text { the task cannot be accomplished using a non-privileged account. }\end{array}$ \\
\hline
\end{tabular}

21 http://dx.doi.org/10.6028/NIST.SP.800-53r4 


\begin{tabular}{|c|c|}
\hline $\begin{array}{l}\text { AC-6, LEAST PRIVILEGE, control } \\
\text { enhancement \#3 }\end{array}$ & $\begin{array}{l}\text { SSH authorized keys providing root or other privileged level access } \\
\text { should be approved and documented before they are provisioned. } \\
\text { Approved authorized keys should specify the command they authorize to } \\
\text { be executed. } \\
\text { An inventory of approved authorized keys and trust relationships should } \\
\text { be maintained, documenting the rationale for each authorized key. }\end{array}$ \\
\hline $\begin{array}{l}\text { AC-6, LEAST PRIVILEGE, control } \\
\text { enhancement \#4 }\end{array}$ & $\begin{array}{l}\text { Unauthorized users should not be given access to private keys that grant } \\
\text { access to privileged accounts. }\end{array}$ \\
\hline $\begin{array}{l}\text { AC-6, LEAST PRIVILEGE, control } \\
\text { enhancement \#5 }\end{array}$ & $\begin{array}{l}\text { Organizations should ensure that non-organizational users cannot obtain } \\
\text { copies of private keys and that any such copies are rendered inoperative } \\
\text { by regular key rotation. }\end{array}$ \\
\hline $\begin{array}{l}\text { AC-6, LEAST PRIVILEGE, control } \\
\text { enhancement \#7 }\end{array}$ & $\begin{array}{l}\text { Privileges granted by SSH keys should be reviewed and adjusted to } \\
\text { reflect organizational/business needs. } \\
\text { Inappropriate, unneeded, or unused authorized keys should be removed } \\
\text { and command and source restrictions added appropriately. }\end{array}$ \\
\hline $\begin{array}{l}\text { AC-6, LEAST PRIVILEGE, control } \\
\text { enhancement } \# 9\end{array}$ & $\begin{array}{l}\text { Encrypted SSH connections accessing privileged accounts should be } \\
\text { audited. }\end{array}$ \\
\hline $\begin{array}{l}\text { AC-6, LEAST PRIVILEGE, control } \\
\text { enhancement } \# 10\end{array}$ & $\begin{array}{l}\text { Authorized keys files should be locked down to prevent users from adding } \\
\text { their own or other public keys to privileged accounts without formal } \\
\text { provisioning or approvals. }\end{array}$ \\
\hline AC-17, REMOTE ACCESS & $\begin{array}{l}\text { Policy should state whether to allow SSH key-based remote access, and } \\
\text { if so, state requirements for key rotation and how to prevent copying of } \\
\text { private keys (e.g., key must be stored on smartcard). Remote access } \\
\text { should be expressly authorized (not provisioned by individual users), } \\
\text { which implies locking down keys. }\end{array}$ \\
\hline $\begin{array}{l}\text { AC-17, REMOTE ACCESS, control } \\
\text { enhancement \#2 }\end{array}$ & $\begin{array}{l}\text { SSH provides such mechanisms. Host key management should be } \\
\text { required for preventing man-in-the-middle attacks. }\end{array}$ \\
\hline $\begin{array}{l}\text { AC-17, REMOTE ACCESS, control } \\
\text { enhancement \#4 }\end{array}$ & $\begin{array}{l}\text { The purpose of each authorized key granting access to root or other } \\
\text { privileged accounts should be documented prior to provisioning access } \\
\text { and such should be limited to [the organization-defined needs]. }\end{array}$ \\
\hline $\begin{array}{l}\text { AC-20, USE OF EXTERNAL } \\
\text { INFORMATION SYSTEMS }\end{array}$ & $\begin{array}{l}\text { SSH user keys can be used for external access. Terms and conditions } \\
\text { should address 1) whether and in what direction is key-based access } \\
\text { allowed; 2) whether command restrictions are required; 3) whether } \\
\text { privileged external access is permitted; 4) what can be done within each } \\
\text { external connection (e.g., is port forwarding allowed); and 5) is DLP } \\
\text { required for content. }\end{array}$ \\
\hline $\begin{array}{l}\text { AU-3, CONTENT OF AUDIT RECORDS, } \\
\text { control enhancement } \# 1\end{array}$ & $\begin{array}{l}\text { Enhanced auditing of SSH should be enabled to track the usage of keys } \\
\text { and provide an audit trail of which source user (and client) is using keys to } \\
\text { connect to the destination user. }\end{array}$ \\
\hline CA-2, SECURITY ASSESSMENTS & $\begin{array}{l}\text { Many organizations have more SSH user key based authenticators } \\
\text { enabling access than they have interactive accounts. Therefore, SSH } \\
\text { user key based access should be addressed in the security assessment } \\
\text { plan and included in the security assessment. }\end{array}$ \\
\hline
\end{tabular}




\begin{tabular}{|c|c|}
\hline CA-3, SYSTEM INTERCONNECTIONS & $\begin{array}{l}\text { SSH user keys define permanent trust relationships that interconnect } \\
\text { information systems (possibly allowing anyone gaining privileged access } \\
\text { to one information system to gain unrestricted access to the other } \\
\text { information system). SSH key-based trust relationships between } \\
\text { information systems must be 1) expressly authorized; } 2 \text { ) documented } \\
\text { (including security requirements, such as key rotation); and 3) periodically } \\
\text { reviewed. }\end{array}$ \\
\hline $\begin{array}{l}\text { CA-5, PLAN OF ACTION AND } \\
\text { MILESTONES }\end{array}$ & $\begin{array}{l}\text { Since unmanaged SSH user keys can undermine so many other security } \\
\text { controls, the action plan should include corrective actions to address SSH } \\
\text { user key-based security weaknesses and deficiencies noted during the } \\
\text { assessment. }\end{array}$ \\
\hline CA-7, CONTINUOUS MONITORING & $\begin{array}{l}\text { SSH-based access should be regularly analyzed as part of a continuous } \\
\text { monitoring program to detect unauthorized authorized keys configured by } \\
\text { users or system administrators (necessary for maintaining and enforcing } \\
\text { security authorizations in dynamic environments). }\end{array}$ \\
\hline $\begin{array}{l}\text { CA-10, INTERNAL SYSTEM } \\
\text { CONNECTIONS }\end{array}$ & $\begin{array}{l}\text { Intra-system SSH connections should be properly authorized and } \\
\text { documented. Typically such connections are configured using SSH user } \\
\text { keys, so authorization and documentation should be required before } \\
\text { provisioning authorized keys. }\end{array}$ \\
\hline $\begin{array}{l}\text { CM-3, CONFIGURATION CHANGE } \\
\text { CONTROL }\end{array}$ & $\begin{array}{l}\text { SSH keys and SSH configuration files are security-sensitive configuration } \\
\text { information, and their misconfiguration, modification, or unauthorized } \\
\text { disclosure may expose servers to unintended access or vulnerabilities. } \\
\text { Thus, changes to them should be reviewed, documented, and audited. }\end{array}$ \\
\hline $\begin{array}{l}\text { CM-5, ACCESS RESTRICTIONS FOR } \\
\text { CHANGE }\end{array}$ & $\begin{array}{l}\text { Provisioning and configuring authenticators for automated access to an } \\
\text { account should be a controlled, judged decision, balancing the need for } \\
\text { access against the risks and must include consideration of the level of } \\
\text { access required. Users and system administrators should not be able to } \\
\text { modify SSH keys and configuration files without oversight. }\end{array}$ \\
\hline CM-6, CONFIGURATION SETTINGS & $\begin{array}{l}\text { Standard configurations for sshd_config settings (including } \\
\text { AuthorizedKeysFile and PermitRootLogin parameters) should be defined } \\
\text { and applied to all SSH servers. SSH key configurations should be } \\
\text { documented and approved. Changes should be monitored. }\end{array}$ \\
\hline CP-2, CONTINGENCY PLAN & $\begin{array}{l}\text { SSH user keys are frequently used in systems that copy data to disaster } \\
\text { recovery sites and implement switching operations to use disaster } \\
\text { recovery sites. Poorly managed SSH trust relationships (e.g., ones } \\
\text { without command restrictions) may be used to spread an attack between } \\
\text { sites. The contingency plan should not rely on switching operations to } \\
\text { disaster recovery sites if a trust relationship without a command restriction } \\
\text { permits access from one site to another, allowing attacks and malware to } \\
\text { spread between sites. }\end{array}$ \\
\hline $\begin{array}{l}\text { IA-2, IDENTIFICATION AND } \\
\text { AUTHENTICATION (ORGANIZATIONAL } \\
\text { USERS) }\end{array}$ & $\begin{array}{l}\text { SSH identity keys should be associated with an individual user. } \\
\text { Policy should prohibit users from sharing private keys, using another } \\
\text { user's private key, or copying/moving a private key to another system. } \\
\text { Monitoring of key usage should be performed to detect instances where } \\
\text { keys are being shared by multiple users, and compromised/shared keys } \\
\text { should be rotated. }\end{array}$ \\
\hline $\begin{array}{l}\text { IA-3, DEVICE IDENTIFICATION AND } \\
\text { AUTHENTICATION }\end{array}$ & Unique SSH host keys should be used for every host running SSH. \\
\hline
\end{tabular}




\begin{tabular}{|c|c|}
\hline IA-5, AUTHENTICATOR MANAGEMENT & $\begin{array}{l}\text { SSH user keys (particularly identity keys) are authenticators. SSH identity } \\
\text { key creation and corresponding authorized key installation should involve } \\
\text { administrative procedures. } \\
\text { Lifetime of SSH user keys should be limited. They should be periodically } \\
\text { rotated. } \\
\text { Policy should prohibit users from sharing private keys, using another } \\
\text { user's private key, or copying/moving a private key to another system. } \\
\text { Continuous monitoring and regular key rotation should be used to enforce } \\
\text { the policy. } \\
\text { Shared accounts on jump servers are group/role accounts, and any } \\
\text { private keys stored on such accounts (for access from the jump server to } \\
\text { end hosts) should be changed when a user's access to the shared } \\
\text { account is terminated (effectively, membership in the group of people with } \\
\text { access to the account is terminated). }\end{array}$ \\
\hline $\begin{array}{l}\text { IA-5, AUTHENTICATOR } \\
\text { MANAGEMENT, control enhancement } \\
\text { \#7 }\end{array}$ & $\begin{array}{l}\text { Password-based automated access using passwords coded in scripts or } \\
\text { binaries should be prohibited. }\end{array}$ \\
\hline $\begin{array}{l}\text { IA-8, IDENTIFICATION AND } \\
\text { AUTHENTICATION (NON- } \\
\text { ORGANIZATIONAL USERS) }\end{array}$ & $\begin{array}{l}\text { The same SSH private key (identity key) should not be used for more than } \\
\text { one user. }\end{array}$ \\
\hline MA-2, CONTROLLED MAINTENANCE & $\begin{array}{l}\text { SSH configurations and authorized keys should be checked after } \\
\text { maintenance to ensure they are still properly configured and functioning } \\
\text { (e.g., as part of continuous monitoring). }\end{array}$ \\
\hline PL-2, SYSTEM SECURITY PLAN & $\begin{array}{l}\text { Automated access should be considered when defining and enforcing the } \\
\text { authorization boundary. } \\
\text { A system that can execute commands on another system (e.g., using } \\
\text { SSH keys) should be considered to have at least the same security } \\
\text { categorization as the system(s) it can access. } \\
\text { Trust relations and automated SSH connections with/to other systems } \\
\text { should be documented in the security plan. }\end{array}$ \\
\hline $\begin{array}{l}\text { PL-8, INFORMATION SECURITY } \\
\text { ARCHITECTURE }\end{array}$ & $\begin{array}{l}\text { Automated access and SSH key-based access must be considered in the } \\
\text { information security architecture. Given that many organizations have } \\
\text { more SSH user keys granting access than they have PKI tokens or } \\
\text { passwords, they cannot be ignored when developing the information } \\
\text { security architecture. }\end{array}$ \\
\hline PS-4, PERSONNEL TERMINATION & $\begin{array}{l}\text { SSH keys are authenticators that may be associated with an individual. } \\
\text { Some individuals may also have had access to authenticators used for } \\
\text { shared accounts or privileged accounts (e.g., SSH private keys stored on } \\
\text { servers). } \\
\text { Any authenticators associated with an individual should be terminated } \\
\text { upon the individual's employment - this means at minimum removing the } \\
\text { related authorized keys from all servers. } \\
\text { Any authenticators that the individual may have had access to should be } \\
\text { rotated (i.e., changed) to ensure that copies of the authenticators do not } \\
\text { remain operable. }\end{array}$ \\
\hline PS-6, ACCESS AGREEMENTS & $\begin{array}{l}\text { Access should not be provisioned without proper access agreement. This } \\
\text { is one more reason why users or system administrators provisioning key- } \\
\text { based access to themselves or others without proper approvals is not } \\
\text { acceptable. }\end{array}$ \\
\hline
\end{tabular}




\begin{tabular}{|l|l|}
\hline RA-3, RISK ASSESSMENT & $\begin{array}{l}\text { Risk assessment should consider attack propagation risk: if one system is } \\
\text { compromised, an attack could spread to other systems using SSH keys } \\
\text { (especially if command restrictions are not used), possibly even to backup } \\
\text { systems and disaster recovery sites. }\end{array}$ \\
$\begin{array}{ll}\text { The potential impact of an SSH key compromise should be part of the risk } \\
\text { assessment process. }\end{array}$ \\
$\begin{array}{l}\text { Authorized keys grant access to an information system. It is impossible to } \\
\text { assess the risk facing an information system without knowing and having } \\
\text { control of who can access the system and what other systems the system } \\
\text { under inspection can access without further authentication. }\end{array}$ \\
\hline ES-12, CRYPTOGRAPHIC KEY & $\begin{array}{l}\text { SSH keys should be rotated and reissued on a regular basis. } \\
\text { SSH user keys should be created only through a controlled provisioning } \\
\text { process. }\end{array}$ \\
\hline SC-23, SESSION AUTHENTICITY & $\begin{array}{l}\text { Unique host keys should be used for every SSH server, as that prevents } \\
\text { man-in-the-middle attacks. }\end{array}$ \\
\hline $\begin{array}{l}\text { SI-4, INFORMATION SYSTEM } \\
\text { MONITORING }\end{array}$ & $\begin{array}{l}\text { SSH connections using key-based authentication should be monitored } \\
\text { and checked against authorized connections to detect unauthorized use } \\
\text { of authorized keys (e.g., using copied identity keys) in real time. }\end{array}$ \\
\hline
\end{tabular}




\section{Appendix B-Cybersecurity Framework Subcategory Mapping}

This appendix lists the Framework for Improving Critical Infrastructure Cybersecurity Version 1.0 22 subcategories that are most pertinent for securing SSH-based automated access management. Next to each subcategory is an explanation of its implications particular to SSH-based automated access management.

\begin{tabular}{|c|c|}
\hline $\begin{array}{l}\text { Cybersecurity Framework } \\
\text { Subcategory }\end{array}$ & SSH Implications \\
\hline $\begin{array}{l}\text { ID.AM-3: Organizational } \\
\text { communication and data flows are } \\
\text { mapped }\end{array}$ & $\begin{array}{l}\text { SSH user keys define permanent trust relationships that interconnect } \\
\text { information systems (possibly allowing anyone gaining privileged access to } \\
\text { one information system to gain unrestricted access to the other information } \\
\text { system). }\end{array}$ \\
\hline $\begin{array}{l}\text { ID.RA-5: Threats, vulnerabilities, } \\
\text { likelihoods, and impacts are used to } \\
\text { determine risk }\end{array}$ & $\begin{array}{l}\text { Risk assessment should consider attack propagation risk: if one system is } \\
\text { compromised, an attack could spread to other systems using SSH keys. The } \\
\text { potential impact of an SSH key compromise should be part of the risk } \\
\text { assessment process. } \\
\text { Authorized keys grant access to an information system. It is impossible to } \\
\text { assess the risk facing an information system without knowing and having } \\
\text { control of who can access the system and what other systems the system } \\
\text { under inspection can access without further authentication. }\end{array}$ \\
\hline $\begin{array}{l}\text { PR.AC-1: Identities and credentials } \\
\text { are managed for authorized devices } \\
\text { and users }\end{array}$ & $\begin{array}{l}\text { SSH user keys authorize access to the information system and specify } \\
\text { privileges for access. SSH identity keys should be associated with an } \\
\text { individual user. Unique SSH host keys should be used for every host } \\
\text { running SSH. }\end{array}$ \\
\hline PR.AC-3: Remote access is managed & $\begin{array}{l}\text { SSH can provide key-based remote access. Remote access should be } \\
\text { expressly authorized (not provisioned by individual users). There should be } \\
\text { requirements for remote access, such as requirements for key rotation and } \\
\text { preventing copying of private keys. }\end{array}$ \\
\hline $\begin{array}{l}\text { PR.AC-4: Access permissions are } \\
\text { managed, incorporating the principles } \\
\text { of least privilege and separation of } \\
\text { duties }\end{array}$ & $\begin{array}{l}\text { Command restrictions should be configured for SSH authorized keys } \\
\text { whenever possible to limit what can be done with the keys. Key-based } \\
\text { access to root accounts should be limited to cases where it is necessary to } \\
\text { accomplish the task. Only those authorized keys that are necessary for } \\
\text { accomplishing the assigned tasks should be configured. }\end{array}$ \\
\hline PR.DS-2: Data-in-transit is protected & $\begin{array}{l}\text { SSH connections using key-based authentication protect the confidentiality } \\
\text { and integrity of data in transit. }\end{array}$ \\
\hline $\begin{array}{l}\text { PR.DS-6: Integrity checking } \\
\text { mechanisms are used to verify } \\
\text { software, firmware, and information } \\
\text { integrity }\end{array}$ & $\begin{array}{l}\text { Authorized keys and other SSH configuration files installed without proper } \\
\text { authorization should be detected (e.g., using continuous monitoring tools). }\end{array}$ \\
\hline $\begin{array}{l}\text { PR.IP-3: Configuration change control } \\
\text { processes are in place }\end{array}$ & $\begin{array}{l}\text { SSH keys and SSH configuration files are security-sensitive configuration } \\
\text { information, and their misconfiguration, modification, or unauthorized } \\
\text { disclosure may expose servers to unintended access or vulnerabilities. } \\
\text { Thus, changes to them should be reviewed, documented, and audited. }\end{array}$ \\
\hline $\begin{array}{l}\text { PR.PT-1: Audit/log records are } \\
\text { determined, documented, } \\
\text { implemented, and reviewed in } \\
\text { accordance with policy }\end{array}$ & $\begin{array}{l}\text { Enhanced auditing of SSH should be enabled to track the usage of keys and } \\
\text { provide an audit trail of which source user (and client) is using keys to } \\
\text { connect to the destination user. }\end{array}$ \\
\hline
\end{tabular}

22 http://www.nist.gov/manuscript-publication-search.cfm?pub_id=915385 


\begin{tabular}{|l|l|}
\hline \multicolumn{1}{|c|}{$\begin{array}{c}\text { Cybersecurity Framework } \\
\text { Subcategory }\end{array}$} & \multicolumn{1}{c|}{ SSH Implications } \\
\hline $\begin{array}{l}\text { PR.PT-3: Access to systems and } \\
\text { assets is controlled, incorporating the } \\
\text { principle of least functionality }\end{array}$ & $\begin{array}{l}\text { SSH user keys define permanent trust relationships that interconnect } \\
\text { information systems (possibly allowing anyone gaining privileged access to } \\
\text { one information system to gain unrestricted access to the other information } \\
\text { system). SSH key-based trust relationships between information systems } \\
\text { must be 1) expressly authorized; 2) documented (including security } \\
\text { requirements, such as key rotation); and 3) periodically reviewed. }\end{array}$ \\
\hline $\begin{array}{l}\text { DE.CM-7: Monitoring for unauthorized } \\
\text { personnel, connections, devices, and } \\
\text { software is performed }\end{array}$ & $\begin{array}{l}\text { SSH-based access should be regularly analyzed as part of a continuous } \\
\text { monitoring program to detect unauthorized authorized keys configured by } \\
\text { users or system administrators (necessary for maintaining and enforcing } \\
\text { security authorizations in dynamic environments). }\end{array}$ \\
\hline
\end{tabular}




\section{Appendix C-SSH Key Management Tool Selection}

The following areas should be considered when selecting and procuring tools for managing SSH keys:

Enterprise Platform: Support for the functionality required for enterprise deployments, including:

o Load balancing and failover so that failure of a single component does not affect system availability and operation.

o Geographic distribution of components for disaster recovery.

o Effective operation across firewalls and network boundaries so that all network zones can be managed.

o Secure architecture, including configurable authentication (password, smartcard, multifactor), granular access controls, and secure logging of all management and operational events.

o Third party security assessments and code reviews.

o Ability to scale to sustainably manage the current number of SSH assets and planned growth, including potential acquisitions.

o Application programming interfaces (APIs) to programmatically access all relevant functionality of the system, including configuration, writing and retrieving data, triggering provisioning operations, discovery, reporting, etc. Support for multiple languages and remote access to APIs. Permissions must be enforced to restrict functional and data access via APIs to authorized individuals or processes.

o Ability to integrate with enterprise infrastructure and systems, including enterprise directories, identity management, ticketing, SIEM systems, CMDBs, hardware security modules (HSMs), workflow, etc.

o Ability to optionally manage all cryptographic assets (e.g., certificates and symmetric keys) from same platform.

Discovery: Automated discovery of all SSH keys and configuration information, including the ability to:

o Perform discovery with or without an agent of all SSH key information, including identity keys, authorized keys, authorized key restrictions, configuration information, file permissions, and log information regarding key usage. Discover and identify encrypted identity keys.

o Identify systems that have not yet been scanned by analyzing discovered data, such as orphan keys (authorized keys for which no corresponding identity keys have been found) and logs that show access to or from other systems on which scanning has not yet been performed.

o Optionally network discover SSH servers or import data about SSH servers from other systems (e.g., vulnerability scanning) that are already performing network scans.

o Support discovery of multiple types of systems (e.g., Unix, Linux, Windows, appliances) and vendor configurations/formats to ensure comprehensive, organization-wide discovery.

o Use SSH configuration files to determine the expected location of identity and authorized keys, and scan those locations or configure scanning of "non-standard" locations. 
o Effectively scan mounted volumes (e.g., Network File System [NFS] or Common Internet File System [CIFS]) and provide an accurate representation of resulting logical key configuration.

o Configure scanning schedules uniquely for different groups of assets, providing options for different intervals (hourly, daily, weekly, monthly, quarterly, annually) and times of day. Tune scanning parameters to ensure that scan processing does not impact the operation and performance of applications and processes on production systems.

- Inventory: Cataloging of all SSH assets, including the ability to:

o Centrally catalog all relevant and discovered information about SSH assets, including keys, key locations (file paths, server and client addresses), associated account information, and configuration information (e.g., authorized key restrictions), as well as information about "external" keys used in connections that span outside the organization and may be held by other organizations or independent users.

o Optionally manually add keys to the inventory via user interfaces or bulk import to accommodate situations where automated discovery is not possible.

o Set policies for critical attributes — such as key length, allowed algorithms, source and command restrictions, and cryptoperiods—and identify keys that are out of compliance with those policies.

o Assign asset "owners” and delegate administration of SSH assets through the assignment of granular permissions and access controls.

o Assign approvers to SSH assets to ensure that access to each asset has been approved by the appropriate people.

o Configure rules for automatically organizing and grouping discovered assets based on attributes of those assets to facilitate delegation.

o Add custom metadata fields to SSH assets to enable the collection of data required by internal processes (e.g., business owner, department, environment, cost center, application ID).

o Automatically send inventory information to other systems on a scheduled basis (e.g., sending trust relationship information to a privileged access management system so that SSH-based entitlements may be reviewed, or updating a CMDB for central tracking of system configuration information).

- Analysis and Reporting: Visual and programmatic tools for ongoing analysis, review, and potential identification of anomalies and vulnerabilities not caught by automated monitoring processes, including:

o Ability to view, analyze, and report on inventory contents, including sorting, filtering, and export.

o Identifying potential security issues, including backdoor keys and duplicated or shared private keys, and boundary crossing (e.g., development to production).

o Automatically mapping trust relationships for review and analysis. Identification of trust relationship configuration that may enable pivoting.

o Dashboard views that provide a high-level view of critical statistics (e.g., deployed key lengths, algorithms, duplicate keys, potential backdoor keys, assets that are out of compliance) and ability to drill down to view applicable keys. Customization of dashboard views to allow the most important information to be shown. 
o Automated generation of reports that provide detailed information on a variety of critical statistics (e.g., key lengths, algorithms, old keys, trust relationships):

- Ability to customize contents and formatting of reports.

- Ability to set delivery schedules for reports and configure intended recipients for reports.

- Ability to configure individualized reports to include only the responsible group or individual's assets within the reports.

- Provisioning: Adding, configuring, removing, and rotating SSH keys, including the ability to:

o Set one or more policies to require specific configuration options during provisioning and management operations (e.g., key lengths, algorithms, cryptoperiods, source and command restrictions, environment crossing).

o Manage lifecycle of identity and authorized keys, including generation of identity keys, adding and removing authorized keys, and integration with identity management and other systems to automatically terminate access (remove authorized keys) on change of role or termination.

o Automatically rotate identity and authorized keys prior to end of configured cryptoperiods.

o Support configuration and management of encrypted identity keys.

o Rollback key rotations in case an issue is encountered with new keys.

o Designate external keys (e.g., an authorized key that is configured to allow access from an identity key held by another organization).

o Restrict permissions on SSH key and configuration files (e.g., authorized keys) or relocate authorized keys to a secure location to prevent unauthorized changes.

o Provide a user portal or other automated mechanism for requesting new identity keys or new access (via authorized keys).

o Configure and require approvals (dual control) for key management operations, including key generation, adding/removing authorized keys, rotations, etc. Log approvals and associated information. Allow for assignment of one or more approvers or groups of approvers.

o Automatically replace large numbers of SSH keys (e.g., in case of a broad system compromise).

o Support deployed SSH products for provisioning and management operations.

o Integrate with ticketing and change control systems to trigger, approve, and track provisioning operations.

Continuous Monitoring: Provide continuous monitoring of SSH keys, including:

o Verification of deployed configuration and keys against approved configuration.

o Configurable notifications when unauthorized keys or configurations are detected.

o Option to automatically remediate out-of-compliance situations (e.g., rollback changed keys, deleted authorized keys).

o Track and monitor key usage and identify unused keys. 
o Ensure regular reviews of SSH-based entitlements are performed and reapproved.

o Identify keys and configuration that are out of compliance with configured policies.

o Tuning and scheduling frequency of monitoring processes.

- Notifications and Alerting: Ability to automatically send notifications/alerts based on specific events, including the ability to:

o Configure which events for which to send notifications (e.g., detected unauthorized keys, errors, keys older than cryptoperiods) and set thresholds where applicable.

o Configure different target recipients for specific events and groups of assets.

o Escalate alerts if action is not taken within a configurable period of time.

o Configure methods of notification, including email, Simple Network Management Protocol (SNMP), syslog, or direct integration with SIEM, ticketing, or other systems.

- Auditing: Ability for auditors to perform reviews of the overall SSH environment and SSH management system, including:

o Logging of all relevant operations and management actions within the management solution to enable forensic-level analysis.

o Ability to view, sort, and filter log events.

o Read-only access to reports and dashboard functionality to enable high-level review and drilldown on all relevant aspects of the SSH environment.

Given that labor costs of the remediation project and the cost of ongoing key provisioning form the bulk of total costs around managing automated SSH-based access, it is important to consider these aspects of a project and select a product that minimizes the total cost of the project—-this may not necessarily be the lowest cost product.

A well-designed product can produce operational cost savings and pay for itself by reducing manual labor previously expended on SSH key provisioning, termination, and rotation-and improve operational flexibility by reducing lead times in provisioning — in addition to meeting the security requirements driving the project.

If the organization does not have sufficient understanding of SSH keys, automated access management, and the remediation process in-house, use of outside expertise to assist in the process may be warranted. 


\section{Appendix D-Acronyms and Abbreviations}

Selected acronyms and abbreviations used in this publication are defined below.

\begin{tabular}{ll} 
AD & Active Directory \\
AES & Advanced Encryption Standard \\
BIOS & Basic Input/Output System \\
CCM & Cipher Block Chaining-Message Authentication Code \\
CIFS & Common Internet File System \\
CMAC & Cipher-Based Message Authentication Code \\
DLP & Data Loss Prevention \\
DSA & Digital Signature Algorithm \\
FIPS & Federal Information Processing Standard \\
FISMA & Federal Information Security Management Act \\
HMAC & Hash Message Authentication Code \\
HSM & Hardware Security Module \\
IT & Information Technology \\
ITL & Information Technology Laboratory \\
LDAP & Lightweight Directory Access Protocol \\
MAC & Message Authentication Code \\
NAT & Network Address Translation \\
NFS & Network File System \\
NIST & National Institute of Standards and Technology \\
OMB & Office of Management and Budget \\
OS & Operating System \\
PIV & Personal Identity Verification \\
RFC & Request for Comment \\
SFTP & Secure File Transfer Protocol \\
SHA & Secure Hash Algorithm \\
SIEM & Security Information and Event Management \\
SP & Special Publication \\
SSH & Secure Shell \\
TGT & Ticket Granting Ticket \\
VPN & Virtual Private Network \\
& \\
\hline & \\
TPN &
\end{tabular}


Appendix E-Glossary

Selected terms used in this publication are defined below.

Authorized Key A public key that has been configured as authorizing access to an account by anyone capable of using the corresponding private key (identity key) in the $\mathrm{SSH}$ protocol. An authorized key may be configured with certain restrictions, most notably a forced command and a source restriction.

Authorized Keys File

Automated Access

\section{Automated Process}

External Key

Fingerprint

Forced Command

Host Key

Identity Key
The file associated with a specific account where one or more authorized keys and optional restrictions are stored. Each account for which public key authentication is allowed on an SSH server has a unique authorized keys file.

Access to a computer by an automated process without an interactive user, generally machine-to-machine access. Automated access is often triggered from scripts or schedulers, e.g., by executing an SSH client or a file transfer application. Many programs may also use automated access using SSH internally, including many privileged access management systems and systems management tools.

An application, script, or management system that leverages SSH to execute commands or transfer data to/from another system.

An authorized key that is used from outside the organization (or outside the environment considered for SSH user key management purposes), or an identity key that is used for authenticating to outside the organization (or outside the environment considered for SSH user key management purposes). Key rotation can break external keys, and therefore it must be ensured that the other side of trust relationships involving external keys is also properly updated as part of rotation. Alternatively, rotation of external keys may be prevented, but that is not a sustainable solution long-term.

A hash value of a (public) key encoded into a string (e.g., into hexadecimal). Several fingerprint formats are in use by different SSH implementations.

A restriction configured for an authorized key that prevents executing commands other than the specified command when logging in using the key. In some SSH implementations, forced command can be configured by using a "command=" restriction in an authorized keys file.

A public key used for authenticating a host in the SSH protocol to hosts that want to communicate with it (each host also generally has its own private host key). Some hosts may have more than one host key (e.g., one for each algorithm). Host keys are used for authenticating hosts (machines) themselves, not users or accounts, whereas identity keys and authorized keys relate to authenticating users/accounts and authorizing access to accounts on hosts.

A private key that is used for authentication in the SSH protocol; grants access to the accounts for which the corresponding public key has been configured as an authorized key. 


\section{Interactive User}

Key

Key Rotation

Known Hosts File

Passphrase

Pivot

Public and Private Key

Source Restriction
A person who uses an SSH client to access one or more SSH servers, typically to perform administrative operations, transfer data, or access applications.

A cryptographic key. In this document, keys generally refer to public key cryptography key pairs used for authentication of users and/or machines (using digital signatures). Examples include identity key and authorized keys. The SSH protocol also uses host keys that are used for authenticating SSH servers to SSH clients connecting them.

Changing the key, i.e., replacing it by a new key. The places that use the key or keys derived from it (e.g., authorized keys derived from an identity key, legitimate copies of the identity key, or certificates granted for a key) typically need to be correspondingly updated. With SSH user keys, it means replacing an identity key by a newly generated key and updating authorized keys correspondingly.

A file associated with a specific account that contains one or more host keys. Each host key is associated with an SSH server address (IP or hostname) so that the server can be authenticated when a connection is initiated. The user or administrator who makes the first connection to an SSH server is responsible for verifying that the host key presented by that server is the actual key (not a rogue key) before it gets placed in the known hosts file.

A password used to protect an identity key. After entered by a user or administrator, a passphrase is mathematically converted into large number which serves as a key that is used to encrypt the identity key. In order to decrypt the identity, the passphrase must be entered again so that the same key can be regenerated for decryption.

The act of an attacker moving from one compromised system to one or more other systems within the same or other organizations. Pivoting is fundamental to the success of advanced persistent threat (APT) attacks. SSH trust relationships may more readily allow an attacker to pivot.

Public and private keys are two very large numbers that (through advanced mathematics) have a unique relationship, whereby information encrypted with one number (key) can only be decrypted with the other number (key) and vice versa. In order to leverage this characteristic for security operations, once two numbers are mathematically selected (generated), one is kept secret (private key) and the other is shared (public key). The holder of the private key can then authenticate themselves to another party who has the public key. Alternatively, a public key may be used by one party to send a confidential message to the holder of the corresponding private key. With SSH, the identity key is a private key and authorized keys are public keys.

A restriction configured for an authorized key that limits the IP addresses or host names from which login using the key may take place. In some SSH implementations, source restrictions can be configured by using a "from=" restriction in an authorized keys file. 
SSH Client

SSH Key

SSH Server

Trust Relationship

User Key
The software implementation that enables a user or an automated process to remotely access an SSH server. An SSH client is responsible for reliably performing all of the operations necessary to ensure a secure connection, including generating identity keys, prompting users to verify host keys, authenticating and establishing encrypted connections with SSH servers, prompting users for credentials, performing public key authentication, etc.

A term that is generally used to refer to an identity and authorized keys. The term may also be occasionally used to refer to host or server private keys.

A software implementation that enables SSH access to a system from SSH clients. SSH server may be included with an operating system or appliance or may be add-on software. An SSH server is typically a complex set of software modules responsible for a broad number of tasks, including enforcing configured SSH settings, authenticating users, limiting access to certain users and groups, ensuring secure connections, interfacing with other systems (e.g., PAM and Kerberos), performing file transfers, etc.

The access relationship that is granted by an authorized key in an account on one system (server) and a corresponding identity key in an account on another system (client). Once deployed, these two keys establish a persistent trust relationship between the two accounts/systems that enables ongoing access.

A key that is used for granting access to a user account via the SSH protocol (as opposed to a host key, which does not grant access to anything but serves to authenticate a host). Both authorized keys and identity keys are user keys. A user key is the equivalent of an access token. 


\section{Appendix F-References}

References for the publication are listed below.

[ID-SSH] Ylonen, T., Kent, G., and Souppaya, M., "Managing SSH Keys for Automated Access Current Recommended Practice”, Internet-Draft, April 2013.

[RFC4251] Ylonen, T. and Lonvick, C., “The Secure Shell (SSH) Protocol Architecture”, RFC 4251, January 2006.

[RFC4252] Ylonen, T. and Lonvick, C., “The Secure Shell (SSH) Authentication Protocol”, RFC 4252, January 2006.

[RFC4253] Ylonen, T. and Lonvick, C., “The Secure Shell (SSH) Transport Layer Protocol”, RFC 4253, January 2006.

[RFC4254] Ylonen, T. and Lonvick, C., “The Secure Shell (SSH) Connection Protocol”, RFC 4254, January 2006. 